\title{
On positive harmonic functions in cones and cylinders
}

\author{
Alano Ancona
}

\begin{abstract}
We first consider a question raised by Alexander Eremenko and show that if $\Omega$ is an arbitrary connected open cone in $\mathbb{R}^{d}$, then any two positive harmonic functions in $\Omega$ that vanish on $\partial \Omega$ must be proportional - an already known fact when $\Omega$ has a Lipschitz basis or more generally a John basis. It is also shown however that when $d \geq 4$, there can be more than one Martin point at infinity for the cone though non-tangential convergence to the canonical Martin point at infinity always holds. In contrast, when $d \leq 3$, the Martin point at infinity is unique for every cone. These properties connected with the dimension are related to wellknown results of $\mathrm{M}$. Cranston and T. R. McConnell about the lifetime of conditioned Brownian motions in planar domains and also to subsequent results by R. Bañuelos and B. Davis. We also investigate the nature of the Martin points arising at infinity as well as the effects on the Martin boundary resulting from the existence of John cuts in the basis of the cone or from other regularity assumptions. The main results together with their proofs extend to cylinders $\mathcal{C}_{Y}(\Sigma)=\mathbb{R} \times \Sigma$ (where $\Sigma$ is a relatively compact region of a manifold $M$ ), equipped with a suitable second order elliptic operator.
\end{abstract}

\section{Introduction and main results}

We consider the cone $\mathcal{C}_{o}(\Sigma)$ of $\mathbb{R}^{d}, d \geq 2$, generated by a region $\Sigma$ of the unit sphere $S_{d-1}$, i.e., $\mathcal{C}_{o}(\Sigma)=\{r \omega ; r>0, \omega \in \Sigma\}$, and study positive harmonic functions in $\mathcal{C}_{o}(\Sigma)$ (where $\mathcal{C}_{o}$ is for cone). Recently Alexander Eremenko asked whether it is always true that any two such functions that moreover vanish on $\partial \mathcal{C}_{o}(\Sigma)$ must be proportional. Our first main result, Theorem 1.1 below (see also Theorem 2.13), answers this question by the positive. A generalization to a large class of cylinders is described in Section 7.

Mathematics Subject Classification (2010): 31C35, 60J45, 60J60, 45C05.

Keywords: Cone, cylinder, Martin boundary, harmonic function, heat kernel, elliptic second order operator. 
To deal with non necessarily Dirichlet-regular $\Sigma$, we say, following a usual convention, that a function $w$ in $\Sigma$ vanishes on the open subset $T$ of $\partial \Sigma$ (or, more precisely, that $w$ vanishes in the weak sense on $T$ ) if $w$ is bounded in a neighborhood of each $\xi \in T$ and if

$$
A:=\left\{\xi \in T ; \limsup _{\Sigma \ni x \rightarrow \xi}|w(x)|>0\right\}
$$

is polar in $S_{d-1}$. By definition, $A \subset S_{d-1}$ is polar in $S_{d-1}$ if for each $\xi \in A$ there is a chart of $S_{d-1}, \chi: V \rightarrow W \subset \mathbb{R}^{d-1}, V \ni \xi$, such that $\chi(V \cap A)$ is polar in $\mathbb{R}^{d-1}$. Note that $A$ is polar in $S_{d-1}$ if and only if $\{t \xi ; \xi \in A, t>0\}$ is polar in $\mathbb{R}^{d}$.

For a function $w$ defined in a region $\Omega$ of $\mathbb{R}^{d}$, the relation $w=0$ on $T \subset \partial \Omega$ is defined similarly. If $w: \Omega \rightarrow \mathbb{R}$ is harmonic with respect to a second order uniformly elliptic operator in divergence form with bounded measurable coefficients in $\Omega$ and if $w=0$ in an open subset $W$ of $\partial \Omega$ then $\lim _{x \rightarrow \xi} w(x)=0$ for every Dirichletregular boundary point $\xi \in W$.

Theorem 1.1. The nonnegative harmonic functions in $\mathcal{C}_{o}(\Sigma)$ which vanish (in the weak sense) on the boundary of $\mathcal{C}_{o}(\Sigma)$ are the functions $h$ in the form

$$
h(r \omega)=c r^{\alpha_{\Sigma}} \varphi_{0}(\omega), \quad \omega \in \Sigma, r>0
$$

where $c$ is a nonnegative constant, $\alpha_{\Sigma}=\frac{-(d-2)+\sqrt{(d-2)^{2}+4 \lambda_{1}(\Sigma)}}{2}$ and $\varphi_{0}$ is a positive solution of $\Delta_{S_{d-1}} \varphi_{0}+\lambda_{1}(\Sigma) \varphi_{0}=0$ in $\Sigma$.

Here $\Delta_{S_{d-1}}$ is the spherical Laplacian - denoted also by $\Delta_{S}$ in the rest of the paper - and $\lambda_{1}(\Sigma)$ (later denoted $\lambda_{1}$ ) is the first eigenvalue of the opposite of the Dirichlet Laplacian in $\Sigma$. As well-known $\lambda_{1}(\Sigma)$ coincides with the Raleigh constant of $\Sigma$, i.e. $\lambda_{1}(\Sigma)=\inf \left\{\int|\nabla u|^{2} d \sigma ; u \in C_{c}^{1}(\Sigma), \int|u|^{2} d \sigma_{S_{d-1}} \geq 1\right\}$ (where $\sigma=\sigma_{S_{d-1}}$ is the standard Riemannian spherical measure in $\left.S_{d-1}\right)$ and $\lambda_{1}(\Sigma)$ is $>0$ if and only if $S_{d-1} \backslash \Sigma$ is not polar in $S_{d-1}$ (see e.g. [20]). In this case, $\frac{1}{\lambda_{1}(\Sigma)}$ is also the largest eigenvalue of the (nonnegative self-adjoint compact) Green's operator in $L^{2}\left(\Sigma ; \sigma_{S_{d-1}}\right), \varphi \mapsto G(\varphi)=\left(-\Delta_{S}\right)^{-1}(\varphi)$. It is known (see e.g. [5]) that $\lambda_{1}(\Sigma)$ is also the greatest real $\lambda$ for which there is a positive $\left(\Delta_{S}+\lambda I\right)$-superharmonic function in $\Sigma$ (distinct from the constant $+\infty$ ). For $\lambda=\lambda_{1}(\Sigma)$ such a function is unique - up to multiplication by a constant - and there is a unique positive solution $\varphi_{0} \in H_{0}^{1}(\Sigma)$ of $\Delta_{S} \varphi_{0}+\lambda_{1}(\Sigma) \varphi_{0}=0$ with $\left\|\varphi_{0}\right\|_{L^{2}(\Sigma)}=1$. In particular $\varphi_{0}=0$ in $\partial \Sigma$. Since, as well-known, the function $H_{0}(x)=r^{\alpha_{\Sigma}} \varphi_{0}(\omega)(r=|x|, \omega=x /|x|)$ is harmonic, Theorem 1.1 means that any two positive harmonic functions in $\mathcal{C}_{o}(\Sigma)$ vanishing on $\partial \mathcal{C}_{o}(\Sigma)$ are proportional. Note also that Theorem 1.1 implies that $H_{0}$ is a positive minimal harmonic function in $\mathcal{C}_{o}(\Sigma)$.

Section 2 is devoted to a proof of Theorem 1.1. See Theorem 2.11 and an improvement in Theorem 2.13. It relies in particular on the study of minimal Martin functions arising at infinity in $\mathcal{C}_{0}(\Sigma)$ and the study of the convergence in the Martin topology towards such Martin points (for Martin's theory, see [26], [31], [17], or [5]). 
When $\Sigma$ is sufficiently regular Theorem 1.1 is well-known. See [25] for the NTA case. The recent paper of K. Hirata [23] establishes the result when $\Sigma$ is John. These papers rely on (and provide) Harnack boundary inequalities which do not hold in the general case.

In Section 6 we show that - in contrast with the case where $\Sigma$ is John - another question which might seem at first to be another formulation of A. Eremenko's question has a negative answer for a general $\Sigma$, at least in higher dimensions.

Theorem 1.2. For $d \geq 4$, there exists a domain $\Sigma$ such that the Martin boundary of $\mathcal{C}_{o}(\Sigma)$ contains a one parameter family of minimal points which are limits of sequences $\left\{P_{n}\right\}$ in $\mathcal{C}_{o}(\Sigma)$ going to infinity in $\mathbb{R}^{d}$ (and whose all defining sequences go to infinity in $\mathbb{R}^{d}$ ).

The class of examples provided to prove Theorem 1.2 is strongly related to the construction by Cranston and McConnell of a bounded domain $\mathbb{D}$ in $\mathbb{R}^{3}$ with a positive harmonic function $h$ in $\mathbb{D}$ such that the lifetime of the $h$-Brownian motion is almost surely infinite [14]. As shown in [14] this cannot happen in a bounded planar domain. There is a corresponding result here given by the next statement. The only interesting case is $d=3$.

Theorem 1.3. If $d \leq 3$ and $\Sigma$ is a domain in $S_{d-1}$, every sequence $\left\{P_{n}\right\}$ in $\mathcal{C}_{o}(\Sigma)$ going to infinity in $\mathbb{R}^{d}$ converges in the Martin topology towards the canonical Martin function $\mathrm{H}_{0}$.

The proof is given in Section 5 and relies on a result of Bañuelos and Davis [10]. A similar proof shows that for all $d \geq 2$, every sequence $\left\{P_{n}\right\}$ in $\mathcal{C}_{o}(\Sigma)$ going nontangentially to infinity in $\mathcal{C}_{o}(\Sigma)$ converges in the Martin topology to $H_{0}$. See Theorem 3.1 and its proof in Section 5. Extensions based on [8] to $d \geq 4$ of Theorem 1.3 are also mentioned there.

In Section 6.4 we supplement Theorem 1.2 by showing that for $d \geq 4, \mathcal{C}_{0}(\Sigma)$ may admit plenty of minimal as well as non-minimal Martin points associated to sequences $\left\{P_{n}\right\}$ in $\mathcal{C}_{o}(\Sigma)$ going to infinity in $\mathbb{R}^{d}$ (and whose all defining sequences go to infinity in $\left.\mathbb{R}^{d}\right)$. The corresponding Martin functions are of the form $H(x)=$ $r^{\alpha} \psi\left(\frac{x}{|x|}\right)$ with $-\frac{d-2}{2}<\alpha<\alpha_{\Sigma}$. This supplementary construction is based on the following fact (see Theorem 4.6) proved also in Section 6.4 and valid for all $d \geq 2$.

Proposition 1.4. If $\Sigma$ contains a hemisphere $\Sigma_{+}$of $S_{d-1}$, then every sequence $\left\{P_{n}\right\}$ such that $\frac{P_{n}}{\left|P_{n}\right|} \in \Sigma_{+}$and $\left|P_{n}\right| \rightarrow+\infty$ converges towards the canonical Martin point $H_{0}$.

In Section 4, some implications on the Martin boundary that follow from regularity conditions are observed. For example, if $M$ is a closed John regular subset of $\Sigma$ (see Section 4 ) then every sequence $\left\{P_{n}\right\}$ in $\mathcal{C}_{o}(M)$ such that $\left|P_{n}\right| \rightarrow \infty$ converges towards the canonical minimal point $H_{0}$. This generalizes Hirata's main result in [23].

To prove the above mentioned results, it seems more convenient (and natural) to work with a cylinder model of $\mathcal{C}_{o}(\Sigma)$ given by $\mathbb{R} \times \Sigma$ equipped with a suitable 
elliptic operator $L$. See Section 2.1. We note here that there is a large literature dealing with Martin's boundaries of product structures (e.g., [27], [19], [29], [30] or [21]). We also refer to [16] [24] [37] [38] and the references there for various other results about harmonic or subharmonic functions in cones and cylinders, in particular results about the Dirichlet problem or the Phragmen-Lindelöf property.

Almost all the results (and their proofs) extend to the framework of a cylinder $\mathcal{C}_{Y}(\Sigma):=\mathbb{R} \times \Sigma$ where $\Sigma$ is a relatively compact region in a manifold $M$, the cylinder being equipped with an elliptic operator $L$ in the form $L=\left(\partial_{t} \circ \partial_{t}+\beta \partial_{t}\right) \oplus \mathcal{L}$ where $\beta \in \mathbb{R}, \partial_{t}$ is differentiation with respect to the first variable and $\mathcal{L}$ is a second order uniformly elliptic operator in $M$. See Section 7.

\section{Proof of Theorem 1.1}

We will assume once for all that $S_{d-1} \backslash \Sigma$ is non polar in $S_{d-1}$. Otherwise, by a standard extension theorem and the Liouville property, positive harmonic functions in $\mathcal{C}_{0}(\Sigma)$ are constant, $\lambda_{1}(\Sigma)=0$ and Theorem 1.1 is obvious in this case. Thus, in what follows, 0 is a Dirichlet-regular boundary point for $\mathcal{C}_{o}(\Sigma)$-using e.g. Wiener's test - and $\lim _{x \rightarrow 0} h(x)=0$ for the functions $h$ under consideration in Theorem 1.1.

\subsection{Reduction and some preliminary remarks}

As a first step, using a simple change of coordinates we reduce the study of positive harmonic functions in the cone $\mathcal{C}_{o}(\Sigma)$ to the study of positive solutions in the cylinder $\mathcal{C}_{Y}(\Sigma)=\mathbb{R} \times \Sigma$ of some natural elliptic operator - the notation $\mathcal{C}_{Y}(\Sigma)$ will be used all along the paper-. Recall first the following classical expression of the Laplacian in polar coordinates: if $f$ is $C^{2}$-smooth in the open set $U$ of $\mathbb{R}^{d}, d \geq 2$,

$$
\Delta f(x)=\frac{\partial^{2} g}{\partial r^{2}}(r, \omega)+\frac{d-1}{r} \frac{\partial g}{\partial r}(r, \omega)+\frac{1}{r^{2}}\left(\Delta_{S}\right)_{\omega} g(r, \omega)
$$

for $x=r \omega,(r, \omega) \in \tilde{U}:=\left\{(r, \omega) \in \mathbb{R}_{+}^{*} \times S_{d-1} ; r \omega \in U\right\}$ and $g(r, \omega)=f(r \omega)$ for $(r, \omega) \in \tilde{U}$. (Recall that $\Delta_{S}$ is the Laplace-Beltrami operator on the Riemannian sphere $\left.S_{d-1}\right)$.

Consider then the change of coordinates: $\Phi(r, \omega)=(\log (r), \omega)$, or $\Phi^{-1}(u, \omega)$ $=\left(e^{u}, \omega\right)$. Obviously $\Phi$ defines a $C^{\infty}$ diffeomorphism of $\mathbb{R}_{+}^{*} \times \Sigma$ onto the cylinder $\mathcal{C}_{Y}(\Sigma)=\mathbb{R} \times \Sigma$. By an elementary calculation, if $g \in C^{2}\left(\mathbb{R}_{+}^{*} \times \Sigma\right)$ and $h=g \circ \Phi^{-1}$, we have

$$
\begin{aligned}
\left(\Delta_{S}\right)_{\omega} g(r, \omega)+(d-1) r \frac{\partial g}{\partial r}(r, \omega)+r^{2} \frac{\partial^{2} g}{\partial r^{2}}= & \left(\Delta_{S}\right)_{\omega} h(u, \omega)+\cdots \\
& +\frac{\partial^{2} h}{\partial u^{2}}(u, \omega)+(d-2) \frac{\partial h}{\partial u}(u, \omega) .
\end{aligned}
$$

Using these two formulas we are reduced to the study of the Martin boundary of the cylindrical region $\mathcal{C}_{Y}(\Sigma)=\mathbb{R} \times \Sigma$ of the manifold $X:=\mathbb{R} \times S_{d-1}$ with respect to the second order elliptic operator $L:=\Delta_{S}+(d-2) \frac{\partial}{\partial u}+\frac{\partial^{2}}{\partial u^{2}}$ (where $u$ denotes the $\mathbb{R}$-component). 
There is a well known explicit (and elementary) expression of the heat semigroup $\left\{Q_{t}\right\}_{t \geq 0}$ associated to the component of $L$ acting in $\mathbb{R}$, i.e., $L_{\mathbb{R}}=\partial_{u}^{2}+$ $(d-2) \partial_{u}$. By heat semi-group associated to $L_{\mathbb{R}}$ we will mean that for each $\varphi \in$ $C_{0}^{+}(\mathbb{R})$ the function $f:(t, x) \mapsto Q_{t}(\varphi)(x)$ is the minimal positive solution of the Cauchy problem: $\partial_{t} f(t, x)=\partial_{x}^{2} f(t, x)+(d-2) \partial_{x} f(t, x)$ for $t>0$ and $f(0, x)=$ $\varphi(x), x \in \mathbb{R}$.

Lemma 2.1. For $t>0$ the kernel $Q_{t}$ on $\mathbb{R}$ is given by the convolution with the density

$$
q_{t}(u)=\frac{1}{\sqrt{4 \pi t}} \exp \left(-\frac{(u+(d-2) t)^{2}}{4 t}\right), \quad u \in \mathbb{R} .
$$

This means that $Q_{t}(\varphi)(u)=\int_{\mathbb{R}} q_{t}(u-v) \varphi(v) d v$ for $\varphi \in C_{0}(\mathbb{R})$. The product structure of $\mathbb{R} \times \Sigma$ is exploited through the next standard fact.

Lemma 2.2. In $\mathbb{R} \times \Sigma$, the heat semi-group $\left\{H_{t}\right\}_{t>0}$ associated to $L=\frac{\partial^{2}}{\partial u^{2}}+$ $(d-2) \frac{\partial}{\partial u}+\Delta_{S}$ is given by the densities

$$
h(t ; u, x ; v, y)=q_{t}(u-v) \pi_{t}(x, y), \quad x, y \in \Sigma, \quad u, v \in \mathbb{R}, t>0 .
$$

Here $\pi_{t}, t>0$, is the standard heat kernel density in $\Sigma$ (with respect to $\Delta_{S}$, the usual Riemannian measure $\sigma_{S}$ in $S_{d-1}$ and the Dirichlet boundary condition).

In other words

$$
H_{t}(\varphi)(u, x)=\int_{-\infty}^{\infty} \int_{\Sigma} h(t ; u, x ; v, y) \varphi(v, y) d v d \sigma_{S}(y)
$$

if $\varphi \in C_{0}(\mathbb{R} \times \Sigma ; \mathbb{R})$ and $(u, x) \in \mathcal{C}_{Y}(\Sigma)$.

\subsection{Some inequalities satisfied by Green's function and their conse- quences}

The Green's function with pole $(v, y)$ and with respect to $L$ in $\mathbb{R} \times \Sigma$ (and the measure $\mu(d(u, x))=d u d \sigma(x))$ is the function:

$$
(u, x) \mapsto G(u, x ; v, y)=\int_{0}^{\infty} h(t ; u, x ; v, y) d t, \quad(u, x) \in \mathbb{R} \times \Sigma .
$$

But $h(t ; u, x ; v, y)=\frac{1}{\sqrt{4 \pi t}} e^{-\frac{(u-v+(d-2) t)^{2}}{4 t}} \pi_{t}(x, y)$ and for $\rho>0$ we have

$$
\begin{aligned}
e^{-\frac{(u+\rho-v+(d-2) t)^{2}}{4 t}} & =e^{-\frac{(u-v+(d-2) t)^{2}}{4 t}} e^{-\frac{\rho}{2 t}\left(\frac{\rho}{2}+(d-2) t+u-v\right)} \\
& \geq e^{-(d-2) \rho / 2} e^{-\frac{(u-v+(d-2) t)^{2}}{4 t}}
\end{aligned}
$$

if $v \geq u+\rho / 2$. 
On the other hand, if $\rho>0$, and $v \leq u+\rho / 2$

$$
\begin{aligned}
e^{-\frac{(u+\rho-v+(d-2) t)^{2}}{4 t}} & =e^{-\frac{(u-v+(d-2) t)^{2}}{4 t}} e^{\frac{\rho}{2 t}\left(-\frac{\rho}{2}-(d-2) t+v-u\right)} \\
& \leq e^{-(d-2) \rho / 2} e^{-\frac{(u-v+(d-2) t)^{2}}{4 t}}
\end{aligned}
$$

Upon integrating with respect to $t$ these inequalities, we obtain the next proposition.

Proposition 2.3. The Green's function $G$ for $L$ in $\mathbb{R} \times \Sigma$ satisfies the following relations:

$$
G(u, x ; v, y) \leq e^{(d-2) \rho / 2} G(u+\rho, x ; v, y), \quad \text { for } u, v \in \mathbb{R}, \text { and } x, y \in \Sigma,
$$

if $\rho>0$ and $v \geq \rho / 2+u$. And

$$
G(u, x ; v, y) \geq e^{(d-2) \rho / 2} G(u+\rho, x ; v, y)
$$

if $\rho>0$ and $v \leq \rho / 2+u$.

Moreover, for $x, y \in \Sigma$ and $u, v, v_{0}, v_{1} \in \mathbb{R}$, we have the following symmetry identities:

$$
G\left(v_{0}-u, x ; v_{0}-v, y\right)=e^{(d-2)(u-v)} G\left(v_{1}+u, x ; v_{1}+v, y\right) .
$$

Note that (2.7) follows immediately from (2.3), Lemma 2.2 and (2.4). Of course these identities can be understood in terms of Kelvin's transformation if one returns to the cone $\mathcal{C}_{o}(\Sigma)$ equipped with the usual Laplacian.

The above leads to the following properties of the $L$-Martin functions associated to $L$ in the cylinder $\mathcal{C}_{Y}(\Sigma)$. We choose and fix once for all a reference point $x_{0} \in \Sigma$ and take $\left(0, x_{0}\right)$ as the normalization point for Martin's functions in $\mathcal{C}_{Y}(\Sigma)$. Occasionally, we use the standard notations $\boldsymbol{\Delta}$ (resp. $\boldsymbol{\Delta}_{1}$ ) to denote the Martin boundary (resp. the minimal Martin boundary) of $\left(\mathcal{C}_{Y}(\Sigma), L\right)$, and $\widehat{\mathcal{C}}_{Y}(\Sigma)$ its Martin compactification (ref. [26], [31], [5]).

Proposition 2.4. If $K$ is an L-Martin function in $\mathcal{C}_{Y}(\Sigma)=\mathbb{R} \times \Sigma$ defined by a sequence $\left(v_{j}, y_{j}\right)$ with $v_{j} \rightarrow+\infty, y_{j} \in \Sigma$, (i.e., $K(u, x)=\lim _{j \rightarrow \infty} K_{\left(v_{j}, y_{j}\right)}(u, x)$ where $K_{\left(v_{j}, y_{j}\right)}$ is the Martin kernel $\left.K_{\left(v_{j}, y_{j}\right)}:=G\left(., . ; v_{j}, y_{j}\right) / G\left(0, x_{0} ; v_{j}, y_{j}\right)\right)$ then

$$
K(u+\rho, x) \geq e^{-(d-2) \rho / 2} K(u, x), \quad(u, x) \in \mathbb{R} \times \Sigma
$$

for every $\rho>0$. In particular if $K$ is minimal there exists $\alpha \geq-\frac{d-2}{2}$ such that $K(u, x)=e^{\alpha u} K(0, x),(u, x) \in \mathbb{R} \times \Sigma$, and the function $s(x)=K(0, x)$ is a proper function in $\Sigma$, namely $\Delta_{S}(s)+\lambda s=0$ for $\lambda=\alpha^{2}+(d-2) \alpha$. So

$$
\lambda \geq-\frac{(d-2)^{2}}{4}, \quad \alpha=\frac{2-d+\sqrt{(d-2)^{2}+4 \lambda}}{2}
$$

and $s$ is a minimal positive $\left(\Delta_{S}+\lambda I\right)$-harmonic function in $\Sigma$. 
Proof. The first statement follows from Proposition 2.3 and the definition of Martin functions. If $K$ is minimal, then $(u, x) \mapsto K(u+\rho, x)$ is also $L$-minimal; by (2.8), it follows that $K(u+\rho, x)=c(\rho) K(u, x)$ for some function $c \in C\left(\mathbb{R} ; \mathbb{R}_{+}^{*}\right)$ and all $(\rho, u, x) \in \mathbb{R} \times \mathbb{R} \times \Sigma$ (assuming first $\rho>0$ ). We have $c\left(\rho+\rho^{\prime}\right)=c(\rho) c\left(\rho^{\prime}\right)$ for $\rho, \rho^{\prime} \in \mathbb{R}$ and so $c(\rho)=e^{\alpha \rho}$ for some $\alpha \geq-\frac{d-2}{2}$. The other claims are then immediate.

Note that $\alpha \in\left[\alpha_{0}, \alpha_{\max }\right]$ where

$$
\alpha_{\max }:=\alpha_{\Sigma}=\frac{2-d+\sqrt{(d-2)^{2}+4 \lambda_{1}}}{2} \text { and } \quad \alpha_{0}=-\frac{d-2}{2} .
$$

We set $\alpha_{\min }=\frac{2-d-\sqrt{(d-2)^{2}+4 \lambda_{1}}}{2}$.

Remark 2.5. Similarly if $K$ is an $L$-Martin function related to a sequence $\left(v_{j}, y_{j}\right)$ with $v_{j} \rightarrow-\infty$ and $y_{j} \in \Sigma$, then we have $K(u+\rho, x) \leq e^{-(d-2) \rho / 2} K(u, x)$ for $(u, x) \in \mathbb{R} \times \Sigma$ and $\rho \geq 0$. If $K$ is minimal w.r. to $L$, then $K(u, x)=e^{\alpha u} s(x)$, for some $\alpha \in\left[\alpha_{\min }, \alpha_{0}\right]$ and some $\lambda$-proper function $s$ in $\Sigma$, i.e., $\Delta_{S}(s)+\lambda s=0$ in $\Sigma$. Here

$$
\lambda=\alpha^{2}+(d-2) \alpha \geq-\frac{(d-2)^{2}}{4}, \quad \alpha=\frac{2-d-\sqrt{(d-2)^{2}+4 \lambda}}{2}
$$

and $s$ is $\left(\Delta_{S}+\lambda I\right)$-minimal in $\Sigma$.

Observe that if $\boldsymbol{\Delta}^{+}$(resp. $\boldsymbol{\Delta}^{-}$) is the set of the Martin functions arising from a sequence $\left(v_{j}, y_{j}\right)$ with $\lim v_{j}=+\infty$ (resp. $\left.\lim v_{j}=-\infty\right)$ the identities (2.7) lead to a natural bijection $K \mapsto \tilde{K}$ from $\boldsymbol{\Delta}^{+}$onto $\boldsymbol{\Delta}^{-}$, where $\tilde{K}(u, x):=$ $e^{-(d-2) u} K(-u, x)$.

We note two other observations which complement Proposition 2.4 and Remark 2.5.

Proposition 2.6. If $\zeta \in \Delta$ is a Martin boundary point for $\left(\mathcal{C}_{Y}(\Sigma), L\right)$ which as a point of the Martin compactification is in the closure of both $\left\{(u, x) \in \mathcal{C}_{Y}(\Sigma) ; u \geq \rho\right\}$ and $\left\{(u, x) \in \mathcal{C}_{Y}(\Sigma) ; u \leq \rho^{\prime}\right\}$ for some $\rho, \rho^{\prime} \in \mathbb{R}, \rho^{\prime}<\rho$, then $K_{\zeta}$ is in the form $K_{\zeta}(u, x)=e^{-\frac{d-2}{2} u} f(x)$ where $f$ is a positive solution of $\Delta_{S} f-\frac{(d-2)^{2}}{4} f=0$ in $\Sigma$.

The same conclusion holds for $\zeta \in \boldsymbol{\Delta}_{1}$ if $\zeta$ is in the closure (for the Martin topology) of a set $\mathcal{C}_{Y}^{R}=\{(v, y) ;|v| \leq R, y \in \Sigma\}, R>0$, and if $K_{\zeta}$ is of the form $K_{\zeta}(u, x)=e^{\alpha u} f(x)$. Moreover $f$ is a positive minimal solution of $\Delta_{S} f-\frac{(d-2)^{2}}{4} f=0$ in $\Sigma$.

Proof. In the first case, by the above estimates of Green's function we must have $K_{\zeta}(u, x)=e^{-\frac{d-2}{2} u} f(x)$ for $\rho^{\prime}<u<\rho, x \in \Sigma$, where $f(x)=e^{\frac{d-2}{2} \rho^{\prime}} K_{\zeta}\left(\rho^{\prime}, x\right)=$ $e^{\frac{d-2}{2} \rho} K_{\zeta}(\rho, x)$. Then $f$ is necessarily as in the statement and by the Green's function estimates, we see that $K_{\zeta}(u, x) \geq e^{-\frac{d-2}{2} u} f(x)$ for all $u>\rho^{\prime}$.

But a nonnegative solution $w$ of $L(w)=0$ in a domain $\Omega \subset \mathcal{C}_{Y}(\Sigma)$ that vanishes at some point vanishes everywhere (by Harnack inequalities). Thus $K_{\zeta}(u, x)=$ $e^{-\frac{d-2}{2} u} f(x)$ when $u>\rho^{\prime}$. A similar argument extends this equality to $u<\rho$. 
In the second case, if $\zeta=: \lim \left(v_{j}, y_{j}\right)$, we must also have $\zeta=\lim \left(v_{j}+s, y_{j}\right)$ for every $s \in \mathbb{R}$. So the result follows from the first part of the proposition (the minimality of $f$ being necessary for the minimality of $K_{\zeta}$ ).

\subsection{Positive $L$-harmonic functions vanishing on $\mathbb{R} \times \partial \Sigma$ (a)}

Let $f$ denote a nonnegative $L$-harmonic function in $\mathcal{C}_{Y}(\Sigma):=\mathbb{R} \times \Sigma$ such that $f=0$ on $\mathbb{R} \times \partial \Sigma$ in the weak sense.

By the Martin boundary theory there is a unique integral representation of $f$ in the form

$$
f(u, x)=\int K_{\zeta}(u, x) d \mu_{f}(\zeta), \quad(u, x) \in \mathbb{R} \times \Sigma,
$$

where $\mu_{f}$ is a finite positive Borel measure on the minimal Martin boundary $\boldsymbol{\Delta}_{1}$ of $\left(\mathcal{C}_{Y}(\Sigma), L\right)$ and where $K$ is the $L$-Martin kernel associated to $L$ and the reference point $\left(0, x_{0}\right) \in \Sigma$.

Denote $\boldsymbol{\Delta}_{1}^{\infty}$ the trace on $\boldsymbol{\Delta}_{1}$ of the intersection of the closures (w. r. to Martin's topology) of the sets $X_{N}:=\{(-\infty,-N] \cup[N,+\infty)\} \times \Sigma, N \geq 1$. In other words, a point $\zeta \in \boldsymbol{\Delta}_{1}$ is in $\boldsymbol{\Delta}_{1}^{\infty}$ if and only if there is a sequence of points $\xi_{j}=\left(u_{j}, x_{j}\right) \in$ $\mathbb{R} \times \Sigma$ with $\left|u_{j}\right| \rightarrow+\infty$ converging to $\zeta$. In particular $K_{\zeta}$ is in the form given by either Proposition 2.3 or Remark 2.5.

Lemma 2.7. The measure $\mu_{f}$ is supported by $\boldsymbol{\Delta}_{1}^{\infty}$.

Proof. Let $N \geq 1$. In $\mathcal{C}_{Y}(\Sigma)$, the function $f$ is equal to $R_{f}^{X_{N}}$, its réduite (cf. [11], [13], [17]) on $X_{N}$ (w.r. to $L$ ). This follows from the assumption $f=0$ in $\partial \Sigma \times \mathbb{R}$ and from (a standard extended form of) the maximum principle. So by the Martin boundary theory, the measure $\mu_{f}$ is supported by the set of the points $\zeta \in \boldsymbol{\Delta}_{1}$ such that $X_{N}$ is not minimally thin at $\zeta$. Such a point $\zeta$ is necessarily in the closure of $X_{N}$ in $\widehat{\mathcal{C}}_{Y}(\Sigma)$, the $L$-Martin compactification of $\mathcal{C}_{Y}(\Sigma)$. Whence the result.

Next we will observe a simple condition for $\mu_{f}$ to be concentrated on

$$
\boldsymbol{\Delta}_{1}^{+\infty}:=\boldsymbol{\Delta}_{1} \cap\left\{\zeta \in \widehat{\mathcal{C}}_{Y}(\Sigma) ; \zeta=\lim _{j}\left(u_{j}, x_{j}\right) \text { with } x_{j} \in \Sigma \text { and } u_{j} \rightarrow+\infty\right\} .
$$

Define similarly $\Delta_{1}^{-\infty}$ using sequences $\left\{\left(u_{j}, x_{j}\right)\right\}_{j \geq 1}$ with $\lim u_{j}=-\infty$.

Lemma 2.8. If $f\left(-t, x_{1}\right)=o\left(e^{\frac{d-2}{2} t}\right)$ as $t \rightarrow+\infty$ for some (or all) $x_{1} \in \Sigma$, then the measure $\mu_{f}$ is supported by $\boldsymbol{\Delta}_{1}^{+\infty}$.

Proof. This is because for $u \leq 0$ and $\zeta \in \boldsymbol{\Delta}_{1}^{-\infty}$ we have

$$
K_{\zeta}\left(u, x_{0}\right) \geq e^{-\frac{d-2}{2} u}, \quad K_{\zeta}\left(0, x_{0}\right)=e^{-\frac{d-2}{2} u}
$$

(see Remark 2.5) and

$$
f\left(u, x_{0}\right)=\int K_{\zeta}\left(u, x_{0}\right) d \mu(\zeta) \geq e^{-\frac{d-2}{2} u} \mu\left(\boldsymbol{\Delta}_{1}^{\infty} \backslash \boldsymbol{\Delta}_{1}^{+\infty}\right)=e^{-\frac{d-2}{2} u} \mu\left(\boldsymbol{\Delta}_{1}^{-\infty}\right) .
$$

Thus $\mu\left(\boldsymbol{\Delta}_{1}^{-\infty}\right)=0$. Note the special case where $f\left(t, x_{0}\right)=O(1)$ as $t \rightarrow-\infty$. 


\subsection{End of proof of Theorem 1.1}

In this subsection it is assumed that moreover $f\left(u_{n}, x_{0}\right)=O(1)$ for a real sequence $u_{n} \rightarrow-\infty$, that is $\liminf _{u \rightarrow-\infty} f(u, x)<\infty$ for each $x \in \Sigma$. We will show that $f$ is unique up to a multiplication by a constant and will thus prove Theorem 1.1. Examining the proof, it will be seen later that this assumption on $\liminf _{u \rightarrow-\infty} f\left(u, x_{0}\right)$ can be notably relaxed.

For any given real $a_{0}$ let us denote $\boldsymbol{\Delta}_{1}^{+\infty}\left(a_{0}\right)$ the set of all points $\zeta \in \boldsymbol{\Delta}_{1}^{+\infty}$ such that $K_{\zeta}$ is in the form: $K_{\zeta}(u, x)=\varphi(x) e^{\alpha u},(u, x) \in \mathcal{C}_{Y}(\Sigma)$ for some real $\alpha \geq a_{0}$ and some minimal positive solution of $\Delta_{S} \varphi+\lambda \varphi=0$ in $\Sigma$ where $\lambda=\alpha^{2}+(d-2) \alpha$. We set $\alpha=\alpha(\zeta)$ and $\lambda=\lambda(\zeta)$.

Repeating the argument in Lemma 2.8 we first note the following.

Lemma 2.9. The measure $\mu_{f}$ is concentrated in $\boldsymbol{\Delta}_{1}^{+\infty}(0)$.

Proof. This is again immediate since given $\varepsilon>0$ we have for $u \leq 0$,

$$
f\left(u, x_{0}\right) \geq \int_{\boldsymbol{\Delta}_{1}^{+\infty} \backslash \boldsymbol{\Delta}_{1}^{+\infty}(-\varepsilon)} K_{\zeta}\left(u, x_{0}\right) d \mu_{f}(\zeta) \geq e^{-\varepsilon u_{f}} \mu_{f}\left(\boldsymbol{\Delta}_{1}^{\infty} \backslash \boldsymbol{\Delta}_{1}^{\infty}(-\varepsilon)\right)
$$

and hence $\mu_{f}\left(\boldsymbol{\Delta}_{1}^{+\infty} \backslash \boldsymbol{\Delta}_{1}^{+\infty}(-\varepsilon)\right)=0$ since $\liminf \operatorname{in}_{u \rightarrow-\infty} f\left(u, x_{0}\right)<\infty$.

Recall that $\lambda_{1}=\lambda_{\max }$ is the greatest $\lambda$ for which the equation $\Delta_{S} \varphi+\lambda \varphi=0$ admits a positive solution (or even a positive supersolution) in $\Sigma$.

Lemma 2.10. Set $A=\left\{\zeta \in \Delta_{1}^{+\infty}(0) ; \lambda(\zeta)<\lambda_{\max }(\Sigma)\right\}$. Then $\mu_{f}(A)=0$.

Proof. Assume that $\mu_{f}(A)>0$. Then for some $\lambda_{1}^{\prime}, 0<\lambda_{1}^{\prime}<\lambda_{1}$, the set $A^{\prime}$ of all $\zeta \in \boldsymbol{\Delta}_{1}^{+\infty}(0)$ for which $0 \leq \lambda(\zeta) \leq \lambda_{1}^{\prime}$ has strictly positive measure: $\mu_{f}\left(A^{\prime}\right)>0$. Note that $A^{\prime}$ is the set of the minimal points $\zeta \in \boldsymbol{\Delta}_{1}$ such that for some $\lambda$, $0 \leq \lambda \leq \lambda_{1}^{\prime}$

$$
K_{\zeta}(u, x)=e^{\alpha u} k_{\zeta}^{\lambda}(x),
$$

where $\alpha=\frac{-(d-2)+\sqrt{(d-2)^{2}+4 \lambda}}{2}$ and $k_{\zeta}^{\lambda}$ is a $\Delta_{S}+\lambda I$-minimal function in $\Sigma$.

The function

$$
\varphi(x)=\int_{A^{\prime}} k_{\zeta}^{\lambda}(x) d \mu_{f}(\zeta), \quad x \in \Sigma
$$

is a (strictly) positive $\Delta_{S}$-superharmonic function in $\Sigma$ which satisfies the following:

(i) $\varphi$ vanishes weakly on the boundary of $\Sigma$ (note that $\varphi(x) \leq f(u, x)$ for $x \in \Sigma$, $u \geq 0$ and that by assumption $f=0$ on $\mathbb{R} \times \partial \Sigma$ in the weak sense),

(ii) the positive measure $-\Delta_{S}(\varphi)$ admits (with respect to $\sigma_{S}$ ) the following density: $\psi(x)=\int_{A^{\prime}} \lambda k_{\zeta}^{\lambda}(x) d \mu_{f}(\zeta)$. 
By (i) and a well-known form of the maximum principle $\varphi$ is a potential in $\Sigma$ with respect to the spherical Laplacian $\Delta_{S}$ in $\Sigma$, i.e. its greatest subharmonic minorant $h$ in $\Sigma$ is zero. Indeed there exists a positive $\Delta_{S}$-superharmonic function $s$ going to infinity at each $\zeta \in \partial \Sigma$ where $\varphi$ has a non-zero upper limit since the set of these points $\zeta$ is polar. So by a standard form of the maximum principle $h-\varepsilon s \leq 0$ for each $\varepsilon>0$.

It follows that $\varphi$ is a Green's potential in $\Sigma$ and so using first (ii) and then (2.12) and (ii) again, we get

$$
G_{\Sigma}(\psi)=\varphi \geq \frac{1}{\lambda_{1}^{\prime}} \psi
$$

in $\Sigma$, where $G_{\Sigma}$ denotes the Green's function in $\Sigma$ w.r. to $\Delta_{S}$.

The function $\psi$ is in $L^{2}(\Sigma)$ (it is bounded in $\Sigma$; in fact $\psi$ is also an element of $\left.H_{0}^{1}(\Sigma)\right)$. Thus from $\psi \neq 0$ and $\left\|G_{\Sigma}(\psi)\right\|_{2} \geq \frac{1}{\lambda_{1}^{\prime}}\|\psi\|_{2}$ we infer that $1 / \lambda_{1}=$ $\left\|G_{\Sigma}\right\|_{L\left(L^{2}, L^{2}\right)} \geq 1 / \lambda_{1}^{\prime}$ which is absurd.

On the other hand, we know that every positive eigenfunction $\varphi$ with respect to $\left(\Delta_{S}, \Sigma\right)$ and the eigenvalue $\lambda_{1}=\lambda_{1}(\Sigma)$ is proportional to $\varphi_{0}$ (see e.g. [5]). Moreover $\varphi_{0}$ vanishes at every Dirichlet-regular boundary point $\xi \in \partial_{S} \Sigma$ and so vanishes quasi-everywhere on $\partial_{S} \Sigma$.

Thus we have proved the following, which contains Theorem 1.1. See also Theorem 2.13.

Theorem 2.11. There is a unique positive L-harmonic function $F_{+}$on $\mathcal{C}_{Y}(\Sigma)$ $=\mathbb{R} \times \Sigma$ vanishing (in the weak sense) on the boundary $\mathbb{R} \times \partial \Sigma$ and such that $\liminf \operatorname{in}_{u \rightarrow-\infty} F_{+}\left(u, x_{0}\right)<+\infty$ and $F_{+}\left(0, x_{0}\right)=1$. This function is a minimal Martin function and

$$
F_{+}(u, x)=e^{\alpha_{\max } u} \frac{\varphi_{0}(x)}{\varphi_{0}\left(x_{0}\right)} \quad \text { if } \quad \alpha_{\max }=\frac{2-d+\sqrt{(d-2)^{2}+4 \lambda_{1}}}{2}
$$

(i.e. $\left.\alpha_{\max }=\alpha_{\Sigma}\right)$.

In what follows we keep the notation $F_{+}$for this "canonical" minimal function and let

$$
F_{-}(u, x)=e^{\alpha_{\min } u} \frac{\varphi_{0}(x)}{\varphi_{0}\left(x_{0}\right)}, \quad \alpha_{\min }=\frac{2-d-\sqrt{(d-2)^{2}+4 \lambda_{1}}}{2}
$$

for the similar minimal function related to the end " $u \rightarrow-\infty$ " of the cylinder. We set $\alpha_{0}=-\frac{d-2}{2}$.

In the course of the proof of Theorem 2.11 we have also essentially shown the following facts. Distinguish three classes of minimal Martin functions $K$ w.r. to $\left(\mathcal{C}_{Y}(\Sigma), L\right)$ :

(i) the functions in the form $K_{\zeta}(u, x)=k(x) e^{\alpha u}$ with $0<\left|\alpha-\alpha_{0}\right| \leq \frac{\sqrt{(d-2)^{2}+4 \lambda_{1}}}{2}$,

(ii) those in the form $K_{\zeta}(u, x)=k(x) e^{\alpha_{0} u}$, and

(iii) the class of all other minimal functions. 
Proposition 2.12. If $K_{\zeta}$ is in the third class there is a unique $v_{\zeta} \in \mathbb{R}$ such that $\lim v_{j}=v_{\zeta}$ for any sequence $\left\{\left(v_{j}, y_{j}\right)\right\}$ in $\mathcal{C}_{Y}(\Sigma)$ converging to $\zeta$ and for such a sequence $\lim _{j}\left(w_{j}, y_{j}\right)=\zeta$ whenever $w_{j} \rightarrow v_{\zeta}$ in $\mathbb{R}$. If $K_{\zeta}$ is in the first class, every corresponding sequence $\left\{\left(v_{j}, y_{j}\right)\right\}_{j \geq 1}$ is such that $\lim v_{j}=+\infty$ or $\lim v_{j}=-\infty$ depending whether $\alpha>\alpha_{0}$ or $\alpha<\alpha_{0}$. Finally if $K_{\zeta}$ is in the class (ii) there are sequences $\left(v, y_{j}\right)$ (with a fixed first coordinate) converging to $\zeta$ and for any such sequence $\lim _{j}\left(v_{j}, y_{j}\right)=\zeta$ for every real bounded sequence $\left\{v_{j}\right\}$; moreover there are sequences $\left\{v_{j}\right\}$ such that $\lim v_{j}=+\infty$ and $\lim _{j}\left(v_{j}, y_{j}\right)=\zeta$.

A minimal function in the class (iii) will be said to be of the finite type.

Of course if $\Sigma$ is smooth, the first class reduces to $\left\{F_{+}, F_{-}\right\}$and the second class is empty. We shall see later that there may exist minimal as well as non minimal Martin points $\zeta$ in the form $K_{\zeta}(u, x)=e^{\alpha u} k(x)$, for all $\alpha$ such that $\left|\alpha-\alpha_{0}\right|<\frac{1}{2} \sqrt{(d-2)^{2}+4 \lambda_{1}}$. See 6.4.

Proof. To establish the last claim let $\zeta$ be in the second class. If $v \in \mathbb{R}$ and if $\left(v_{j}, y_{j}\right) \rightarrow \zeta$ then $\left(2 v-v_{j}, y_{j}\right) \rightarrow \zeta$ (by the identities $(2.7)$ with $\left.v_{0}=v_{1}=0\right)$. But a minimal Martin point has a neighborhood basis $\left\{U_{j}\right\}$ in $\widehat{\mathcal{C}}_{Y}(\Sigma)$ with $U_{j} \cap \mathcal{C}_{Y}(\Sigma)$ connected (by the general theory, see e.g. [31], p. 223) and so we can find points $z_{j} \in$ $\Sigma$ with $\left(v, z_{j}\right) \rightarrow \zeta$. If $\left\{v_{j}\right\}$ is bounded it follows at once from the (local) Harnack inequalities and the translation invariance with respect to the first coordinate that $\left(v_{j}, z_{j}\right) \rightarrow \zeta$. It is then obvious that if $v_{j} \rightarrow+\infty$ sufficiently slowly $\left(v_{j}, y_{j}\right) \rightarrow \zeta$.

It also follows immediately, from translation invariance, that if a sequence $\left\{\left(v_{j}, y_{j}\right)\right\}_{j \geq 1}$ converges to a point $\zeta \in \Delta$ then $\lim _{j}\left(w_{j}, y_{j}\right)=\zeta$ for $\left\{w_{j}\right\}$ such that $\left|v_{j}-w_{j}\right| \rightarrow 0$.

\subsection{Positive $L$-harmonic functions vanishing on $\mathbb{R} \times \partial \Sigma$ (b)}

The proof of Lemma 2.10 can be extended so as to use a much weaker assumption on the behavior of $f\left(u, x_{0}\right)$ for $u \rightarrow-\infty$. This leads to a description of the positive $L$-solution in $\mathbb{R} \times \Sigma$ vanishing on $\mathbb{R} \times \partial \Sigma$ which also improves Theorem 2.11.

Theorem 2.13. If $f$ is L-harmonic in $\mathcal{C}_{Y}(\Sigma)$ and vanishes on $\mathbb{R} \times \partial \Sigma$, then $f$ is a linear combination of $F_{+}$and $F_{-}$. Thus if $\liminf _{u \rightarrow-\infty} e^{\alpha_{\min } u} f\left(u, x_{0}\right)=0$ then $f$ is proportional to $F_{+}$.

Proof. Using the Martin disintegration of $F$, we may write $f=a F_{+}+b F_{-}+F_{1}+F_{2}$ with

$$
F_{1}(u, x)=\int_{A} e^{\alpha u} k_{\zeta}(x) d \mu(\zeta), F_{2}(u, x)=\int_{B} e^{\alpha u} k_{\zeta}(x) d \nu(\zeta)
$$

where

$$
\begin{aligned}
& A=\left\{\zeta \in \Delta_{1} ; \exists \alpha_{\zeta}, \alpha_{0} \leq \alpha_{\zeta}<\alpha_{\max } \text { and } k_{\zeta} \in C_{+}(\Sigma) \text { s.t. } K_{\zeta}(u, x) \equiv e^{\alpha_{\zeta} u} k_{\zeta}(x)\right\} \\
& B=\left\{\zeta \in \Delta_{1} ; \exists \alpha_{\zeta}, \alpha_{\min }<\alpha_{\zeta}<\alpha_{0} \text { and } k_{\zeta} \in C_{+}(\Sigma) \text { with } K_{\zeta}(u, x) \equiv e^{\alpha_{\zeta} u} k_{\zeta}(x)\right\}
\end{aligned}
$$

and where $\mu$ and $\nu$ are finite Borel measures supported by $A$ and $B$ respectively. 
We claim that $\mu(A)=0$. If not there exists $\alpha_{1}^{\prime} \in\left(\alpha_{0}, \alpha_{\max }\right)$ such that $\mu\left(A^{\prime}\right)>0$ if $A^{\prime}=\left\{\zeta \in A ; \alpha_{0} \leq \alpha_{\zeta} \leq \alpha_{1}^{\prime}\right\}$ and repeating the argument in Lemma 2.10 we may conclude using now $\varphi(x):=\int_{A^{\prime}} k_{\zeta}(x) d \mu(\zeta)$ and the potential theory w. $\mathrm{r}$. to the operator $\Delta_{S}-\frac{(d-2)^{2}}{4} I=\Delta_{S}-\lambda_{0} I$ in $S_{d-1}$ (in particular the related Green kernel $\left.G_{\Sigma}^{\lambda_{0}}\right)$. Note that $(2.13)$ becomes:

$$
G_{\Sigma}^{\lambda_{0}}(\psi)=\varphi \geq \frac{1}{\left(\lambda_{1}^{\prime}-\lambda_{0}\right)} \psi
$$

where $\lambda_{1}^{\prime}$ is the eigenvalue corresponding to $\alpha^{\prime}$. In the same way (or using the observation after Remark 2.5) it is shown that $F_{2}=0$.

\section{Nontangential convergence to $\boldsymbol{F}_{+}$or $\boldsymbol{H}_{0}$}

The next statement is about how Martin's topology relates to the canonical minimal $F_{+}$in the cylinder $\mathcal{C}_{Y}(\Sigma)$ (or to the minimal $H_{0}$ in the cone $\mathcal{C}_{0}(\Sigma)$ ). It says that nontangential convergence of the current point $(u, x) \in \mathcal{C}_{Y}(\Sigma)$ (resp. $\left.x=r \omega \in \mathcal{C}_{o}(\Sigma)\right)$ to the end " $u=+\infty$ " (or " $r=+\infty$ ") implies its convergence to the canonical Martin point at infinity.

Theorem 3.1. For every sequence $\zeta_{j}:=\left(u_{j}, x_{j}\right)$ in $\mathbb{R} \times \Sigma$ such that $u_{j} \rightarrow+\infty$ and $\left\{x_{j}\right\}$ is relatively compact in $\Sigma$, we have $K_{\zeta_{j}}(u, x) \rightarrow e^{\alpha_{\max } u} \varphi_{0}(x) / \varphi_{0}\left(x_{0}\right)$ (i.e. $\left\{\zeta_{j}\right\}$ converges to the Martin function $F_{+}$). In fact, the following Harnack boundary inequalities hold:

$$
\begin{aligned}
C^{-1} G\left(u, x_{0} ; v, x_{0}\right) G\left(v, x_{0} ; w, x_{0}\right) & \leq G\left(u, x_{0} ; w, x_{0}\right) \\
& \leq C G\left(u, x_{0} ; v, x_{0}\right) G\left(v, x_{0} ; w, x_{0}\right)
\end{aligned}
$$

for $u, v, w \in \mathbb{R}, u+1 \leq v, v+1 \leq w$ and some constant $C=C\left(d, \Sigma, x_{0}\right) \geq 1$. The inequalities obtained by replacing $G$ by its transposed kernel in (3.1) also hold.

A proof will be given at the end of Section 5. We note here that inequalities (3.1) imply by themselves that for $t \rightarrow+\infty$ the point $\left(t, x_{0}\right)$ converges to a minimal point in the Martin boundary (see Théorème 2 of [2] or p. 516 of [3]).

\section{Martin boundary and subsets of $\Sigma$}

In this section we collect some properties of the Martin boundary of $\mathcal{C}_{Y}(\Sigma)$ resulting from regularity conditions on a subset $M$ of $\Sigma$. The results will not be used before Section 6.3.

\subsection{John regular subsets}

Let $M$ be a closed subset of $\Sigma$. For $\eta>0$, let $M_{\eta}:=\left\{x \in \Sigma ; d_{a}(x, M) \leq \eta\right\}$ where $d_{a}(x, y):=\inf \{\operatorname{diam}(C) ; C \subset \Sigma$ connected, $x, y \in C\}\left(S_{d-1}\right.$ is equipped with the usual metric in $\left.\mathbb{R}^{d}\right)$. For $0<c_{0} \leq 1$, we say that $M$ is $c_{0}$-John in $\Sigma$ if there are 
points $A_{1}, \ldots, A_{N}$ in $\Sigma$ such that (i) $d\left(A_{j}, \partial \Sigma\right) \leq c_{0}^{-1} d\left(A_{k}, \partial \Sigma\right)$ for $1 \leq j, k \leq N$, (ii) for $\eta:=c_{0} \max \left\{d\left(A_{j}, \partial \Sigma\right) ; 1 \leq j \leq N\right\}$, each $a \in M_{\eta}$ can be connected to one $A_{j}$ by a $c_{0}$-John arc in $\Sigma$ (see Définition 1.1 and Théorème 5.3 in $[6]$ ). $\left\{A_{j}\right\}_{1 \leq j \leq N}$ is then called a $c_{0}$-admissible set of poles for $M$ (note that $N$ can always be chosen smaller than a constant $\left.N_{0}\left(d, c_{0}\right)\right)$.

The next statement generalizes Hirata's main result in [23]. We rely on Theorem 3.1 and a boundary Harnack principle given in [6] ([1] for $N \leq 2$ ). Note that this statement may be easily reduced to the $N=1$ case.

Theorem 4.1. Let $M$ be a closed and $c_{0}$-John subset of $\Sigma$ with poles $A_{j}, 1 \leq j \leq N$. Then $\lim _{u \rightarrow+\infty}(v, y)=F_{+}$in the Martin topology, uniformly with respect to $y \in M$.

Proof. Denote $K$ the Martin kernel in $\widehat{\mathcal{C}}_{Y}(\Sigma)$ with respect to the normalization point $\left(0, x_{0}\right)$. Applying Théorème 5.3 and Remarque 5.4 in [6] to $M \times[v-1, v+1]$ as a subset of $\mathcal{C}_{Y}(\Sigma)$ (or rather - so as to deal with the classical Laplacian - to the corresponding situation in $\left.\mathcal{C}_{0}(\Sigma)\right)$ we obtain a constant $C=C\left(d, c_{0}\right) \geq 1$ such that

$$
K_{(v, y)}(u, x) \leq C \sum_{j=1}^{N} \frac{K_{(v, y)}\left(v, A_{j}\right)}{K_{\left(v, A_{j}\right)}\left(v, A_{j}^{\prime}\right)} K_{\left(v, A_{j}\right)}(u, x)
$$

whenever $(v, y) \in \mathbb{R} \times M,|v| \geq 1$, and $(u, x) \in \mathcal{C}_{Y}(\Sigma)$ satisfies $|u-v| \geq 1$ (or $\left.d_{a}(x, M) \geq c_{0}\right)$. Here $A_{j}^{\prime}$ is arbitrarily chosen in $\partial B\left(A_{j}, \frac{c_{0}}{100} \operatorname{dist}\left(A_{j} ; S_{d-1} \backslash \Sigma\right)\right)$ and we restrict to $y$ such that $\left|y-A_{j}\right| \geq 2\left|A_{j}-A_{j}^{\prime}\right|$ for all $j$.

By Harnack inequalities,

$$
K_{(v, y)}(u, x) \geq c \frac{K_{(v, y)}\left(v, A_{j}\right)}{K_{\left(v, A_{j}\right)}\left(v, A_{j}^{\prime}\right)} K_{\left(v, A_{j}\right)}(u, x)
$$

when $(u, x) \in \partial B\left(\left(v, A_{j}\right),\left|A_{j}-A_{j}^{\prime}\right|\right)$ and hence - by the maximum principle - also for $(u, x) \in \mathcal{C}_{Y}(\Sigma) \backslash B\left(\left(v, A_{j}\right),\left|A_{j}-A_{j}^{\prime}\right|\right)$. Taking $(u, x)=\left(0, x_{0}\right)$ we see that $c \frac{K_{(v, y)}\left(v, A_{j}\right)}{K_{\left(v, A_{j}\right)}\left(v, A_{j}^{\prime}\right)} \leq 1$. So it follows from (4.1) that

$$
K_{(v, y)}(u, x) \leq C^{\prime} \sum_{j=1}^{N} K_{\left(v, A_{j}\right)}(u, x)
$$

when $(v, y) \in \mathbb{R} \times M,|v| \geq 1$, and $(u, x) \in \mathcal{C}_{Y}(\Sigma)$ satisfies $|u-v| \geq 1$. Since by Theorem $3.1 K_{\left(v, A_{j}\right)} \rightarrow F_{+}$for $j \rightarrow \infty$ and since $F_{+}$is minimal, the result follows.

The proof also yields the following more general statement. Here the results of [6] for John subsets with more than one pole are effectively used.

Theorem 4.1' Let $\left\{M_{n}\right\}$ be a sequence of closed John regular subsets of $\Sigma$ with a common John constant $c_{0}$. Assume that $v_{n} \rightarrow+\infty$ in $\mathbb{R}$ and that $\left(v_{n}, A_{j}^{(n)}\right) \rightarrow$ $\zeta_{j} \in \Delta_{1}, 1 \leq j \leq N$, where for each $n,\left\{A_{j}^{(n)}\right\}_{1 \leq j \leq N}$ is a $c_{0}$-admissible set of poles for $M_{n}$. Then if $y_{n} \in M_{n}$, every Martin cluster function of $\left\{\left(v_{n}, y_{n}\right)\right\}$ is a linear combination of the $K_{\zeta_{j}}$.

In particular if the points $\zeta_{j}$ all coincide with a minimal boundary point $\zeta$ then $\left(v_{n}, y_{n}\right) \rightarrow \zeta$. 


\subsection{John cuts}

Assume now that $M$ is a John regular closed subset of $\Sigma$ and that $\Sigma \backslash M$ is the disjoint union of two open subsets $U_{0}$ and $U_{1}$. Fix $\delta>0$ and set $U_{j}^{\delta}=\left\{x \in U_{j}\right.$; $\left.d_{a}(x, M) \geq \delta\right\}$ and $V_{j}^{\delta}=\mathbb{R} \times U_{j}^{\delta}, j=1,2$. Let $V_{j}=\mathbb{R} \times U_{j}$.

Proposition 4.2. If $h=K_{\mu}$ is the positive superharmonic function in $\mathcal{C}_{Y}(\Sigma)$ generated by a probability measure $\mu$ supported on the closure of $V_{0}$ in the Martin compactification $\widehat{\mathcal{C}}_{Y}(\Sigma)$ and not charging $\left(0, x_{0}\right)$, we have

$$
h(u, x) \leq C\left[F_{+}(u, x)+F_{-}(u, x)\right], \quad(u, x) \in V_{1}^{\delta}
$$

for some constant $C=C\left(\Sigma, M, U_{0}, x_{0}, \delta\right)$.

Proof. We may assume that $x_{0} \in U_{1}^{\delta}$ (using Harnack and changing the reference point) and it suffices to prove (4.3) for each $h=K_{(v, y)}, y \in V_{0}$ with a constant $C>0$ as in the statement. Reducing $h$ on $V_{1}$, it suffices to prove the result for $K_{(v, y)}, y \in M, v \in \mathbb{R}$.

For such a pole $(v, y)$, with say $v>0$, it follows from (4.2) and Theorem 3.1 that for $x \in U_{1}^{\delta}$ such that $d\left(x, A_{j}\right) \geq \frac{1}{2} d\left(A_{j}, \partial \Sigma\right)$ for $j=1, \ldots, N$, (we use the same notations as above)

$$
\begin{aligned}
K_{(v, y)}(u, x) & \leq C^{\prime} \sum_{j=1}^{N} K_{\left(v, A_{j}\right)}(u, x) \\
& \leq C^{\prime \prime} \sum_{j} K_{\left(v, A_{j}\right)}\left(v, A_{j}^{\prime}\right) e^{\alpha_{\max }(u-v)} \varphi_{0}(x) \\
& \leq C^{\prime \prime \prime} e^{\alpha_{\max } u} \varphi_{0}(x) .
\end{aligned}
$$

In the second line we have used the maximum principle (as above in the proof of Theorem 4.1) to compare the positive $L$-harmonic function $e^{\alpha_{\max } u} \varphi_{0}(x)$ with the Green function with pole at $\left(v, A_{j}\right)$. In the last line we have used the inequalities given by Theorem 3.1 which imply that $K_{\left(v, A_{j}\right)}\left(v, A_{j}^{\prime}\right) \simeq K_{\left(v, A_{j}\right)}\left(v-1, A_{j}\right) \simeq$ $F_{+}\left(v-1, A_{j}\right)$. Using the similar inequality for $v \leq 0$ we get the desired conclusion.

Remark 4.3. For $h=K_{(v, y)}, y \in M$, the proof shows that $h(u, x) \leq C\left[F_{+}(u, x)\right.$ $\left.+F_{-}(u, x)\right]$ if $x \in U_{1},|u-v| \geq 1$. If moreover $v \geq 0$, then $h(u, x) \leq C F_{+}(u, x)$, for these points $(u, x)$.

Corollary 4.4. Under the assumptions of Proposition 4.2 two sequences $\left\{\left(v_{j}, y_{j}\right)\right\}$ and $\left\{\left(w_{j}, z_{j}\right)\right\}$ such that $v_{j}, w_{j} \rightarrow+\infty, y_{j} \in U_{0}$ and $z_{j} \in U_{1}$, have at most one common cluster point in $\boldsymbol{\Delta}$ which can only be $F_{+}$.

Corollary 4.5. If $h_{0}$ and $h_{1}$ are positive harmonic in $\mathcal{C}_{Y}(\Sigma)$, if $h_{j}=K_{\mu_{j}}$ with $\mu_{j}$ supported by $\overline{U_{j} \times \mathbb{R}}$ (closure in $\left.\widehat{\mathcal{C}}_{Y}(\Sigma)\right), j=0,1$, if $\lim _{u \rightarrow+\infty} e^{-\alpha_{\max } u}\left(h_{0} \wedge h_{1}\right)\left(u, x_{0}\right)$ $=\lim _{u \rightarrow+\infty} e^{\alpha_{\min } u}\left(h_{0} \wedge h_{1}\right)\left(-u, x_{0}\right)=0$ then $h_{0} \wedge h_{1}$ is a potential (that is, it has no positive L-harmonic minorant). 


\subsection{Inner ball property}

If we have a boundary point $z \in \partial \Sigma$ and an open ball (or cap) $B(a, r) \subset \Sigma$ with $z \in \partial B(a, r), r<2$, the results in [4] (see also [2]) tell us (using Proposition 2.12) that as $v \rightarrow v_{0}$ in $\mathbb{R}$ and $y \rightarrow z$ non-tangentially in $B(a, r)$, the point $(v, y)$ tends to a finite type minimal boundary point $\xi=\xi\left(v_{0} ;(z, a)\right)$ in $\widehat{\mathcal{C}}_{Y}(\Sigma)$. Moreover the minimal $K_{\xi}$ is bounded away from $\left(v_{0}, z\right)$ and vanishes on $\partial \mathcal{C}_{Y}(\Sigma) \backslash\left\{\left(v_{0}, z\right)\right\}$.

There is a parallel statement for the behavior of $(v, y)$ for $v \rightarrow+\infty$. But here the inner ball should be large. This will be used later for an example's construction in Section 6.4.

Theorem 4.6. Assume that $\Sigma$ contains an open hemisphere $\Sigma_{+}$in $S_{d-1}$. Then if $\left\{y_{j}\right\}$ is a sequence in $\Sigma^{+}$and if $v_{j} \rightarrow+\infty$ in $\mathbb{R}$, the sequence $\left(v_{j}, y_{j}\right)$ converges in the Martin compactification of $\mathcal{C}_{Y}(\Sigma)$ to the canonical Martin point $F_{+}$, i.e., $\lim _{j} K_{\left(v_{j}, y_{j}\right)}=F_{+}$.

The proof is deferred to Section 6.4.

\section{Uniqueness of the Martin point at infinity for $d=3$}

We now prove Theorem 1.3 (rather its cylinder version), using in an essential way a result of $\mathrm{R}$. Bañuelos and $\mathrm{B}$. Davis on the heat kernels in planar domains (see [10] and [9]). This result says that given the point $x_{1} \in \Sigma$ there is a $t_{0}>0$ and for each $t \geq t_{0}$ a constant $C(t)>1$ such that $\lim _{t \uparrow \infty} C(t)=1$ and

$$
C(t)^{-1} e^{-\lambda_{1} t} \varphi_{0}\left(x_{1}\right) \varphi_{0}(y) \leq \pi_{t}\left(x_{1}, y\right) \leq C(t) e^{-\lambda_{1} t} \varphi_{0}\left(x_{1}\right) \varphi_{0}(y)
$$

when $t \geq t_{0}$ and $y \in \Sigma$ (see Theorem 1 and Section 4 in [10]). Recall $\left\{\pi_{t}\right\}$ is the heat semi-group generated by the Laplacian in $\Sigma$ and $\varphi_{0}$ is normalized by the condition $\left\|\varphi_{0}\right\|_{L^{2}(\Sigma)}=1$.

Theorem 5.1. If $d=3$, every sequence $\left\{\xi_{j}\right\}_{j \geq 1}=\left\{\left(v_{j}, y_{j}\right)\right\}_{j \geq 1}$, in $\mathcal{C}_{Y}(\Sigma)$ such that $v_{j} \rightarrow+\infty$ converges to $F_{+}$, i.e., $K_{\xi_{j}}(u, x) \rightarrow K_{\zeta_{\infty}}(u, x)$ for $j \rightarrow \infty$ and $K_{\zeta_{\infty}}:=e^{\alpha_{\max } u} \varphi_{0}(x) / \varphi_{0}\left(x_{0}\right)$.

The following simple lemma (valid for all $d \geq 2$ ) deals with times in $\left(0, t_{0}\right]$.

Lemma 5.2. Given $\delta_{0}>0$ and $x_{1} \in \Sigma$, there is a constant $C=C\left(\delta_{0} ; \Sigma, x_{1}\right) \geq 1$ such that

$$
\pi_{t}\left(x_{1}, y\right) \leq C e^{-\lambda_{1} t} \varphi_{0}\left(x_{1}\right) \varphi_{0}(y)
$$

for all $y \in \Sigma$ such that $\left|y-x_{1}\right| \geq \delta_{0}$ and all $t \geq 0$

Assuming as we may that $\delta_{0}<d\left(x_{0}, S_{d-1} \backslash \Sigma\right)$, this is a simple consequence of the parabolic maximum principle applied in the region $\{(x, t) ; t>0, x \in \Sigma\} \backslash$ $\left\{(x, t) ;\left|x-x_{0}\right| \leq \delta_{0}, 0 \leq t \leq 1\right\}$ (the two members of (5.2) are $\left(\partial_{t}-L\right)$-harmonic in $(y, t)$ and the first has by definition minimal growth at infinity in $\left.\Sigma \times \mathbb{R}_{+}\right)$. 
Lemma 5.3. Assume $d=3$ and let $T, \mu>0, x_{1} \in \Sigma$ be given. Then, as $a \rightarrow+\infty$,

$$
\int_{0}^{T} e^{-\frac{a^{2}}{4 t}-\mu t} \pi_{t}\left(x_{1}, y\right) \frac{d t}{\sqrt{t}}=o\left(\int_{T}^{\infty} e^{-\frac{a^{2}}{4 t}-\mu t} \pi_{t}\left(x_{1}, y\right) \frac{d t}{\sqrt{t}}\right)
$$

uniformly in $y \in \Sigma,\left\|y-x_{1}\right\| \geq \delta_{0}$.

Proof. We may assume $T \geq t_{0}$. By (5.1) and Lemma 5.2, it suffices to prove the relation obtained from (5.3) when the terms $\pi_{t}\left(x_{1}, y\right)$ are removed from the integrals. Now,

$$
\int_{0}^{T} e^{-\frac{a^{2}}{4 t}-\mu t} \frac{d t}{\sqrt{t}} \leq \frac{1}{2} \sqrt{T} e^{-\frac{a^{2}}{4 T}} \quad \text { and } \quad \int_{\theta}^{\infty} e^{-\frac{a^{2}}{4 t}-\mu t} \frac{d t}{\sqrt{t}} \geq e^{-\frac{a^{2}}{4 \theta}} \int_{\theta}^{\infty} e^{-\left(\mu+\frac{1}{2}\right) t} d t
$$

for $\theta \geq 1$, where for the last inequality we use the observation that $t \mapsto e^{\frac{t}{2}} / \sqrt{t}$ is increasing in $(1, \infty)$. So

$$
\int_{\theta}^{\infty} e^{-\frac{a^{2}}{4 t}-\mu t} \frac{d t}{\sqrt{t}} \geq \frac{1}{\nu} e^{-\frac{a^{2}}{4 \theta}} e^{-\nu \theta}
$$

where $\nu=\mu+\frac{1}{2}$, and we may conclude since as $a \rightarrow+\infty, \frac{\nu}{2} \sqrt{T} e^{-\frac{a^{2}}{4}\left(\frac{1}{T}-\frac{1}{\theta}\right)+\nu \theta} \rightarrow 0$ for any fixed $\theta>T$.

Proof of Theorem 5.1. Assume as we may that $y_{j} \rightarrow y_{\infty} \in \bar{\Sigma}$. Using the BañuelosDavis Theorem and the above lemma and its proof, we have if $y_{\infty} \neq x_{1}$ and $j \rightarrow \infty$,

$$
\begin{aligned}
\sqrt{4 \pi} G\left(u, x_{1} ; v_{j}, y_{j}\right) & =\int_{0}^{\infty} e^{-\frac{\left(u+(d-2) t-v_{j}\right)^{2}}{4 t}} \pi_{t}\left(x_{1}, y_{j}\right) \frac{d t}{\sqrt{t}} \\
& =e^{\frac{(d-2)\left(v_{j}-u\right)}{2}} \int_{0}^{\infty} e^{-\frac{\left(u-v_{j}\right)^{2}}{4 t}} e^{-\frac{(d-2)^{2}}{4} t} \pi_{t}\left(x_{1}, y_{j}\right) \frac{d t}{\sqrt{t}} \\
& \sim e^{\frac{(d-2)\left(v_{j}-u\right)}{2}} \varphi_{0}\left(x_{1}\right) \varphi_{0}\left(y_{j}\right) \int_{0}^{\infty} e^{-\frac{\left(u-v_{j}\right)^{2}}{4 t}-\left[\frac{(d-2)^{2}}{4}+\lambda_{1}\right] t} \frac{d t}{\sqrt{t}} .
\end{aligned}
$$

Thus, for $x_{1}, x_{2} \in \Sigma$, we see that $G\left(u, x_{2} ; v_{j}, y_{j}\right) / G\left(u, x_{1} ; v_{j}, y_{j}\right) \rightarrow \varphi_{0}\left(x_{2}\right) / \varphi_{0}\left(x_{1}\right)$ for $j \rightarrow \infty$ (assuming first $y_{\infty} \neq x_{1}, y_{\infty} \neq x_{2}$ ). This shows that a cluster function $K$ of the Martin kernels $K_{v_{j}, y_{j}}$, as $j \rightarrow \infty$, is in the form $K(u, x)=g(u) \varphi_{0}(x)$ and hence must be $K=F_{+}$.

In fact,

$$
\int_{0}^{\infty} e^{-\frac{\left(u-v_{j}\right)^{2}}{4 t}-\left[\frac{(d-2)^{2}}{4}+\lambda_{1}\right] t} \frac{d t}{\sqrt{4 \pi t}}=\frac{1}{2 \sqrt{\mu}} e^{-\sqrt{A_{j} \mu}}
$$

where $A_{j}=\left|u-v_{j}\right|^{2}, \mu=\frac{(d-2)^{2}}{4}+\lambda_{1}$ (note that the left member of (5.5) is the Green's function in $\mathbb{R}$ with pole at the origin and with respect to $\mathcal{L}=\frac{d^{2}}{d x^{2}}-\mu$ evaluated at $\left.\sqrt{A_{j}}\right)$. It follows that $G\left(u, x ; v_{j}, y_{j}\right) / G\left(0, x_{0} ; v_{j}, y_{j}\right) \rightarrow F_{+}(u, x)$ when $j \rightarrow \infty$. 
This shows that

$$
G\left(\left(u, x_{1}\right) ;(v, y)\right) \sim \frac{1}{\sqrt{(d-2)^{2}+4 \lambda_{1}}} \varphi_{0}\left(x_{1}\right) \varphi_{0}(y) e^{-\alpha_{\max }(v-u)}
$$

uniformly with respect to $y$, as $v-u \rightarrow+\infty$. Similarly,

$$
G\left(\left(u, x_{1}\right) ;(v, y)\right) \sim \frac{1}{\sqrt{(d-2)^{2}+4 \lambda_{1}}} \varphi_{0}\left(x_{1}\right) \varphi_{0}(y) e^{-\alpha_{\min }(u-v)}
$$

as $v-u \rightarrow-\infty$, uniformly w. r. to $y$.

Proof of Theorem 3.1. The proof of Theorem 3.1 (where $d \geq 2$ ) is completely similar. In fact as well known (see e.g. [12], [33]) for every compact $K \subset \Sigma$, $e^{\lambda_{1} t} \pi_{t}(x, y) \rightarrow \varphi_{0}(x) \varphi_{0}(y)$ as $t \rightarrow+\infty$, uniformly w. r. to $y \in K$. So an obvious adaptation of the above gives the convergence to $F_{+}$. Inequalities (3.1) follow from the fact that now $G\left(u, x_{0} ; 0, x_{0}\right) \sim C^{\prime} e^{\alpha_{\min } u}$ for $u \rightarrow+\infty$.

Remark 5.4. The proof also shows that Theorem 5.1 extends to $d \geq 3$ if the base $\Sigma$ is intrinsically ultracontractive with respect to $\Delta_{S}$ (see [15], [9], [8]). For example, by Bañuelos results in [8] this is the case if for some $p>\frac{n}{2}$ the base $\Sigma$ is $L^{p}$-averaging (that is $\rho_{\Sigma} \in L^{p}(\Sigma)$ where $\rho_{\Sigma}(x)$ is the pseudo-hyperbolic distance to $\left.x_{0}\right)$. See [8] for other examples.

\section{Examples for $d \geq 4$}

In this section we show that for $d \geq 4$ there are cones in $\mathbb{R}^{d}$ with a host of Martin points at infinity. See Sections 6.2 and 6.3 (another example described in Section 6.4 shows that these points can be minimal as well as non minimal). This is closely connected with the existence - established by Cranston and McConnell in [14] - of a bounded domain $D$ in $\mathbb{R}^{3}$ and an $h$-Brownian motion in $D$ that has infinite lifetime. In fact we use a variant of the construction in [14] Section 3.

As before we work with the model of the cone $\mathcal{C}_{0}(\Sigma)$ given by the cylinder $\left(\mathcal{C}_{Y}(\Sigma), L\right)$.

\subsection{Preliminary lemmas}

Fix $\lambda_{0}>0, d \geq 2$, and consider a cap $B=B(a, r):=\{x \in S ;|x-a|<r\}$ in $S:=S_{d-1}, r \leq 1$, with two given points $\xi, \xi^{\prime} \in \partial B$, symmetric in $S$ with respect to $a$. Let

$$
T=B(\xi, r / 100) \cap \partial B, \quad T^{\prime}=B\left(\xi^{\prime}, r / 100\right) \cap \partial B,
$$

and let $M_{B}:=\left\{x \in B ;|x-\xi|=\left|x-\xi^{\prime}\right|\right\}$.

Let $\Omega$ be a region in $S_{d-1}$ such that $B \subset \Omega \subset S_{d-1} \backslash(\partial B \backslash T), \overline{T^{\prime}} \cap \overline{\Omega \backslash B}=\emptyset$. Set $\tilde{\Omega}=\Omega \times \mathbb{R}$. 
Lemma 6.1. Let $v=H_{f}$ solve $\Delta_{S} v-\lambda v-\partial_{t} v=0$ in $\tilde{\Omega}$ and $v(y, t)=f(y, t)$ in $\partial \tilde{\Omega}$ where $f(y, t)$ is bounded measurable in $\partial \tilde{\Omega}$, nondecreasing in $t$ and $f(y, t)=0$ for $y \notin T^{\prime}$. Then, given $\eta \in(0,1)$, there exists $\varepsilon_{1}=\varepsilon_{1}\left(d, \lambda_{0}, \eta\right)>0$ such that for $0<\varepsilon \leq \varepsilon_{1}$ and $0 \leq \lambda \leq \lambda_{0}$,

$$
H_{f}(x, t) \leq \int_{\partial \Omega}\left[\eta f(y, t)+(1-\eta) f\left(y, t-\varepsilon r^{2}\right)\right] d \mu_{x}^{\Omega}(y), \text { for }(x, t) \in M_{B} \times \mathbb{R} .
$$

Moreover $H_{f}(x, t)$ is nondecreasing in $t$. Here $\mu_{x}^{\Omega}$ is the harmonic measure of $x$ in $\Omega$ w. r. to $\Delta_{S}-\lambda I$.

Proof. The last claim follows from the parabolic maximum principle and the translation invariance in $t$ of $\Delta_{S}-\lambda I-\partial_{t}$.

To prove the first, observe that by the monotonicity assumption we may assume that $f(y, s)=\varphi(y)$ for $s>t-\varepsilon r^{2}$ and $f(y, s)=\psi(y)$ when $s \leq t-\varepsilon r^{2}$. Since the inequality is an identity when $f(y, s)$ is independent of $s$ we may assume $\psi=0$ and also that $t=\varepsilon r^{2}$ by time translation invariance. Then,

$$
\int_{\partial \Omega}\left[\eta f\left(y, \varepsilon r^{2}\right)+(1-\eta) f(y, 0)\right] d \mu_{x}^{\Omega}(y)=\eta \Phi(x),
$$

where $\Phi$ solves $\Delta \Phi-\lambda \Phi=0$ in $\Omega$ and $\Phi=f$ on $\partial \Omega$. We want to show that $H_{f}\left(x, \varepsilon r^{2}\right) \leq \eta \Phi(x)$ provided $\varepsilon<1 / 8$ is sufficiently small. Let $N$ be the integer part of $1 /(4 \varepsilon)$ and set $w(x, s)=H_{f}\left(x, s+\varepsilon r^{2}\right)-H_{f}(x, s)$.

By the parabolic Harnack inequalities [28], $C w\left(a, r^{2}(1-k \varepsilon)\right) \geq w\left(a, r^{2} / 2\right)$ for $1 \leq k \leq N$ and a constant $C=C(d)$. Thus, on summing up, $N w\left(a, r^{2} / 2\right) \leq$ $C H_{f}\left(a, r^{2}\right) \leq C \Phi(a)$.

Applying next the parabolic boundary Harnack principle in $\Omega \times \mathbb{R}([18],[22])$ to $w(x, s)$ and $\Phi$ (viewed as functions of $(x, s))$ we obtain

$$
w\left(x, \varepsilon r^{2}\right) \leq c_{1} \frac{w\left(a, r^{2} / 2\right)}{\Phi(a)} \Phi(x) \leq \frac{c_{1} C}{N} \Phi(x)
$$

for $x \in M_{B}$ with $c_{1}=c_{1}\left(C, d, \lambda_{0}\right)$. The result follows.

We will use Lemma 6.1 in conjunction with the following lemma.

Lemma 6.2. Let $\left\{m_{k}\right\}_{1 \leq k \leq N}$ be a finite sequence of probability measures in $\mathbb{R}$ of the form $m_{k}=\frac{1}{2} \delta_{0}+\frac{1}{2} \delta_{-a_{k}}$ for $1 \leq k \leq N$, where $0 \leq a_{k} \leq 1$. Let $L$ and $\varepsilon$ be given positive numbers. There is an $A=A(L, \varepsilon)>0$ such that if $\sum_{k=1}^{N} a_{k} \geq A$, the measure $\nu_{N}=m_{1} * \cdots * m_{N}$ satisfies: $\nu_{N}([-L, 0]) \leq \varepsilon$.

Proof. The probability $\nu_{N}$ is the law of the random variable $Z:=-\sum_{j=1}^{N} a_{j} X_{j}$ if $X_{1}, \ldots, X_{N}$ are independent random variables such that $P\left(X_{j}=0\right)=P\left(X_{j}=1\right)$ $=1 / 2$. For $\beta>0$, we have

$$
\begin{aligned}
P(-Z \leq L) & =P\left(e^{\beta Z} \geq e^{-\beta L}\right) \leq e^{\beta L} E\left(e^{\beta Z}\right) \\
& =e^{\beta L} \prod_{j=1}^{N} E\left(e^{-\beta a_{j} X_{j}}\right)=e^{\beta L} \prod_{j=1}^{N}\left(1-\frac{1-e^{-\beta a_{j}}}{2}\right) .
\end{aligned}
$$


Thus, using $a_{k} \leq 1$, we have

$$
P(Z \geq-L) \leq e^{\beta L} \prod_{j=1}^{N}\left(1-\frac{\beta}{2} e^{-\beta} a_{j}\right) \leq e^{\beta L} \exp \left(-\frac{\beta}{2} e^{-\beta} \sum_{j=1}^{N} a_{j}\right) .
$$

The lemma follows.

\subsection{A class of cylinders}

We now consider domains $\Sigma \subset S_{d-1}, d \geq 4$, similar to examples introduced in [14]: there are disjoint open balls $B_{j}=B\left(x_{j}, r_{j}\right), j \geq 0$, in $S_{d-1}$ such that (i) $\sum_{j \geq 0} r_{j}^{2}=$ $+\infty$, (ii) $B_{j} \subset \Sigma$, (iii) for $N \geq 1, \Sigma \backslash B_{N}$ has two components $\Sigma_{N}^{+}, \Sigma_{N}^{-}$with disjoint closures and $\Sigma_{N}^{-} \supset \bigcup_{j<N} B_{j}, \Sigma_{N}^{+} \supset \bigcup_{j>N} B_{j}$, (iv) there are caps $T_{j}, T_{j}^{\prime}$ in $\partial B_{j}, j \geq 0$, symmetric with respect to $x_{j}$, of radius $\rho_{j} \leq r_{j} / 10$ and such that $\Sigma_{j}^{-} \cap \bar{B}_{j} \subset T_{j}, \Sigma_{j}^{+} \cap \bar{B}_{j} \subset T_{j}^{\prime}$.

Remark 6.3. There is an $\varepsilon_{d}>0$ such that whenever $r_{j}>0, j \geq 1$, satisfy $\sum r_{j}^{2}=\infty$ and $\sum r_{j}^{d-1} \leq \varepsilon_{d}$, there exists a corresponding $\Sigma$ such that moreover: (a) $\left|x_{j}-x_{k}\right| \geq 4 \max \left\{r_{k}, r_{j}\right\}$ for $j \neq k$, (b) the centers $x_{j}$ have a limit $P_{0}$ in $S_{d-1}$ (c) $\Sigma$ is locally Lipschitz in $S_{d-1} \backslash\left\{P_{0}\right\}$ and is Dirichlet-regular in $S_{d-1}$. The proof is left to the reader.

Set $\Sigma_{N}=\Sigma_{N}^{-} \cup B_{N}$ for $N \geq 1$ and fix $\lambda_{0}>0$. Let $k$ be a bounded positive solution of $\Delta k-\lambda k=0, k=0$ in $\partial \Sigma_{N} \backslash T_{N}^{\prime}, 0 \leq \lambda \leq \lambda_{0}$. For $\ell>0$, let $h=h_{\ell}$ solve: $\partial_{t} h(x, t)-\Delta_{x} h(x, t)+\lambda h(x, t)=0$ in $\Sigma_{N} \times \mathbb{R}, h(t, x)=\mathbf{1}_{t \geq-\ell} k(y)$ in $\partial \Sigma_{N} \times \mathbb{R}$.

Proposition 6.4. Let $\ell$ and $\varepsilon$ be positive reals and let $x \in \Sigma_{q}^{-}, 1 \leq q<N$. There is an integer $N_{\Sigma}\left(q, \varepsilon, \ell, \lambda_{0}\right)$ such that whenever $N \geq N_{\Sigma}\left(q, \varepsilon, \ell, \lambda_{0}\right)$,

$$
h_{\ell}(x, 0) \leq \varepsilon k(x) .
$$

Proof. Fix $\eta=1 / 2$ and a corresponding $\varepsilon_{0}>0$ as given by Lemma 6.1 . Let $p \in\{q, q+1, \ldots\}$, let $f(y, t)$ be a bounded Borel function in $T_{p}^{\prime} \times \mathbb{R}$ which is non decreasing in $t$ and let $v=H_{f}$ denote the solution of $\Delta_{S} v-\lambda v-\partial_{t} v=0$ in $\Sigma_{p} \times \mathbb{R}$ with $v=\mathbf{1}_{T_{p}^{\prime} \times \mathbb{R}} f$ on the boundary. We show by induction on $n=p-q$, that

$$
H_{f}(x, t) \leq \int_{T_{p}^{\prime}}\left(\int_{-\infty}^{\infty} f(y, t+s) d \nu_{q, p}(s)\right) d \mu_{x}^{\Sigma_{p}}(y), \quad x \in \Sigma_{q} \backslash B_{q}
$$

where

$$
\nu_{q, p}=\underset{j=q}{\stackrel{p}{\star}}\left(\frac{1}{2} \delta_{0}+\frac{1}{2} \delta_{-\varepsilon_{0} r_{j}^{2}}\right)
$$

and where $\mu_{x}^{\Sigma_{p}}$ is the harmonic measure of $x$ in $\Sigma_{p}$ w.r. to $\Delta_{S}-\lambda I$. Denote $\nu_{j}:=\frac{1}{2} \delta_{0}+\frac{1}{2} \delta_{-\varepsilon_{0} r_{j}^{2}}$. 
For $n=0$ this is Lemma 6.1. Assuming that the property holds for $n-1 \geq 0$ and viewing $H_{f}$ as a solution in $\Sigma_{q} \times \mathbb{R}$ of a Dirichlet problem for $\Delta_{S}-\lambda I-\partial_{t}$ we get by lemma 6.1 and maximum principle that for $x \in \Sigma_{q}^{-}$,

$$
\begin{aligned}
& H_{f}(x, t) \leq \int_{T_{q}^{\prime}}\left(\int_{-\infty}^{+\infty} H_{f}(y, s+t) d \nu_{q}(s)\right) d \mu_{x}^{\Sigma_{q}}(y) \\
& \leq \int_{T_{q}^{\prime}}\left(\int_{-\infty}^{+\infty}\left[\int_{T_{p}^{\prime}} \int_{-\infty}^{+\infty} f(z, s+t+\tau) d \nu_{q+1, p}(\tau) d \mu_{y}^{\Sigma_{p}}(z)\right] d \nu_{q}(s)\right) d \mu_{x}^{\Sigma_{q}(y)} \\
& =\int_{T_{q}^{\prime}}\left(\int_{T_{p}^{\prime}}\left[\int_{-\infty}^{+\infty} \int_{-\infty}^{+\infty} f(z, s+t+\tau) d \nu_{q+1, p}(\tau) d \nu_{q}(s)\right] d \mu_{y}^{\Sigma_{p}}(z)\right) d \mu_{x}^{\Sigma_{q}}(y) \\
& =\int_{T_{q}^{\prime}}\left(\int_{T_{p}^{\prime}}\left[\int_{-\infty}^{+\infty} f(z, t+\theta) d \nu_{q, p}(\theta)\right] d \mu_{y}^{\Sigma_{p}}(z)\right) d \mu_{x}^{\Sigma_{q}}(y) \\
& =\int_{T_{p}^{\prime}}\left[\int_{-\infty}^{+\infty} f(z, t+\theta) d \nu_{q, p}(\theta)\right] d \mu_{x}^{\Sigma_{p}}(z),
\end{aligned}
$$

where we have used in the second line the induction assumption, in the third the fact that integration with respect to $s$ and integration with respect to $z$ commute and - in the last line - the formula

$$
\mu_{x}^{\Sigma_{p}}=\int \mu_{y}^{\Sigma_{p}} d \mu_{x}^{\Sigma_{q}}(y)
$$

(for $x \in \Sigma_{q}^{-}$). This proves (6.3).

From (6.3) it follows that for $x \in \Sigma_{q}^{-}=\Sigma_{q} \backslash B_{q}$,

$$
h_{\ell}(x, 0) \leq \int_{T_{N}^{\prime}}\left(\int_{-\ell}^{0} k(y) d \nu_{q, N}(s)\right) d \mu_{x}^{\Sigma_{N}}(y)=k(x) \nu_{q, N}([-\ell, 0)),
$$

and the proposition follows from lemma 6.2 and the condition $\sum_{j \geq 1} r_{j}^{2}=+\infty$.

We now take for $k$ the Green's function $k=G_{y}^{\lambda}$ in $\Sigma$ with pole at some point $y \in \Sigma \backslash \bar{\Sigma}_{N}$ and with respect to $\Delta_{S}-\lambda I$. It is easily checked that for $-\ell \leq s \leq 0$ and $x \in \Sigma_{N}$,

Recall that

$$
h_{\ell}(s, x) \geq \int_{0}^{\ell-|s|} e^{-\lambda t} \pi_{t}(x, y) d t .
$$

$$
k(x)=\int_{0}^{\infty} e^{-\lambda t} \pi_{t}(x, y) d t
$$

and that the parabolic Green's function with pole at $\left(y_{0}, t_{0}\right)$ in $\Sigma$ (and w.r. to $\left.\Delta_{S}-\lambda-\partial_{t}\right)$ is $\Gamma\left(x, t ; y_{0}, t_{0}\right):(x, t) \mapsto \mathbf{1}_{t>t_{0}} e^{-\lambda\left(t-t_{0}\right)} \pi_{t-t_{0}}\left(x, y_{0}\right)$; thus

$$
u(x, s):=\int_{-\infty}^{+\infty} \mathbf{1}_{\{t>-\ell\}} \Gamma(x, s ; y, t) d t=\int_{0}^{\ell-|s|} e^{-\lambda t} \pi_{t}(x, y) d t
$$

is bounded by $h_{\ell}(x, s)$ in $\Sigma_{N} \cap\{-\ell<s<0\}$ by the parabolic maximum principle. 
Thus, the previous result can be read as follows.

Lemma 6.5. For any sequence $\left\{y_{j}\right\}$ converging in $\Sigma$ to the end $\mathcal{E}$ of $\Sigma$ defined by the cuts $B_{N}$ (a basis for $\mathcal{E}$ is provided by the sets $\Sigma_{N}^{+}$) and every $\lambda \geq 0$, we have

$$
\lim _{j \rightarrow \infty} \frac{\int_{0}^{t_{0}} e^{-\lambda t} \pi_{s}\left(x, y_{j}\right) d s}{\int_{0}^{\infty} e^{-\lambda t} \pi_{s}\left(x, y_{j}\right) d s}=0
$$

for every fixed $t_{0} \geq 0$ and every $x \in \Sigma$.

So,

$$
\lim _{j \rightarrow \infty} \frac{\int_{t_{0}}^{\infty} e^{-\lambda t} \pi_{s}\left(x, y_{j}\right) d s}{\int_{0}^{\infty} e^{-\lambda t} \pi_{s}\left(x, y_{j}\right) d s}=1 \quad \text { for } t_{0} \geq 0, x \in \Sigma \text { and } \lambda \geq 0
$$

Lemma 6.6. Let $\left\{y_{j}\right\}$ be as in Lemma 6.5. For $j \rightarrow \infty$ and for given reals $\rho, \rho^{\prime}$ the ratio

$$
\frac{\int_{0}^{\infty} t^{-\frac{1}{2}} e^{-\frac{(\rho+(d-2) t)^{2}}{4 t}} \pi_{t}\left(x, y_{j}\right) d t}{\int_{0}^{\infty} t^{-\frac{1}{2}} e^{-\frac{\left(\rho^{\prime}+(d-2) t\right)^{2}}{4 t}} \pi_{t}\left(x, y_{j}\right) d t}
$$

converges towards $e^{-\frac{d-2}{2}\left(\rho-\rho^{\prime}\right)}$ for each $x \in \Sigma$.

Proof. We have

$$
\int_{0}^{\infty} t^{-\frac{1}{2}} e^{-\frac{(\rho+(d-2) t)^{2}}{4 t}} \pi_{t}\left(x, y_{j}\right) d t=e^{-\frac{(d-2) \rho}{2}} \int_{0}^{\infty} t^{-\frac{1}{2}} e^{-\frac{\rho^{2}}{4 t}} e^{-t \frac{(d-2)^{2}}{4}} \pi_{t}\left(x, y_{j}\right) d t
$$

If we set $\varphi(t)=t^{-\frac{1}{2}} e^{-\frac{\rho^{2}}{4 t}} e^{-t \frac{(d-2)^{2}}{4}}$, then for every $t_{0}>0$ and for $j \rightarrow \infty$

$$
\int_{0}^{t_{0}} \varphi(t) \pi_{t}\left(x, y_{j}\right) d t=o\left(\int_{t_{0}}^{\infty} \varphi(t) \pi_{t}\left(x, y_{j}\right) d t\right)
$$

In fact, with $A>\frac{(d-2)^{2}}{4}$, we have for $t_{1}>0$ large enough

$$
\int_{t_{1}}^{\infty} \varphi(t) \pi_{t}\left(x, y_{j}\right) d t \geq C\left(t_{1}, A, d\right) \int_{t_{1}}^{\infty} e^{-A t} \pi_{t}\left(x, y_{j}\right) d t
$$

(note that $\frac{\varphi(t)}{\varphi\left(t_{1}\right)} \geq \frac{e^{-A t}}{e^{-A t_{1}}}$ for $t \geq t_{1}$, because $\varphi(t) e^{t A}$ is increasing for $t$ large enough).

On the other hand, for such a fixed $t_{1}$, we have

$$
\int_{0}^{t_{1}} \varphi(t) \pi_{t}\left(x, y_{j}\right) d t \leq C^{\prime}\left(t_{1}, A, \rho, d\right) \int_{0}^{t_{1}} e^{-A t} \pi_{t}\left(x, y_{j}\right) d t
$$

and (6.6) follows by Lemma 6.5. 
Since $e^{-\frac{\rho^{2}}{4 t}} \rightarrow 1$ for $t \rightarrow \infty$ ( $\rho$ being fixed) we see now that as $j \rightarrow+\infty$,

$$
\int_{0}^{\infty} t^{-\frac{1}{2}} e^{-\frac{\rho^{2}}{4 t}} e^{-t \frac{(d-2)^{2}}{4}} \pi_{t}\left(x, y_{j}\right) d t \sim \int_{0}^{\infty} t^{-\frac{1}{2}} e^{-t \frac{(d-2)^{2}}{4}} \pi_{t}\left(x, y_{j}\right) d t
$$

Using also this result for $\rho^{\prime}$ the lemma follows.

\subsection{The Martin boundary of the first example}

Using Lemma 6.6 we get a (partial) description of the Martin boundary of the cylinder $\mathcal{C}_{Y}(\Sigma)=\mathbb{R} \times \Sigma$ with respect to $L:=\partial_{u u}^{2}+\frac{d-2}{2} \partial_{u}+\Delta_{S}$. In particular it will be seen that there are Martin boundary points related to sequences $\left(u_{j}, y_{j}\right) \in \mathcal{C}_{Y}(\Sigma)$ with $\lim u_{j}=+\infty$ and distinct from the canonical point $F_{+}$given by Theorem 2.11 .

Denote $\widetilde{\partial} \Sigma:=\partial \Sigma \cap\left\{\bigcup_{N>1} \partial \Sigma_{N}^{-}\right\}$the set of points in $\partial \Sigma$ "away from the end $\mathcal{E}$ " (see Lemma 6.5). Because $\mathcal{E}$ is defined by a "smooth" system of cuts (the balls $B_{N}$ or the mediators $M_{N}$ of $T_{N}$ and $T_{N}^{\prime}$ in $B_{N}$ ) it follows from standard forms of the boundary Harnack principle (see e.g. [4], [6]) that for $\lambda<\lambda_{1}(\Sigma)$, the end $\mathcal{E}$ is the trace on $\Sigma$ of the neighborhoods system - in the Martin compactification of $\left(\Sigma, \Delta_{S}+\lambda I\right)$ - of a minimal $\Delta_{S}+\lambda I$-harmonic function $k_{\mathcal{E}}^{\lambda}$ (normalized at $x_{0}$ ) which vanishes on $\widetilde{\partial} \Sigma([4]$, Théorème 2.5). define

For $\alpha \in\left[\alpha_{0}, \alpha_{\max }\right)$ (recall $\alpha_{0}:=-\frac{d-2}{2}$ and $\left.\alpha_{\max }:=\frac{-(d-2)+\sqrt{(d-2)^{2}+4 \lambda_{1}(\Sigma)}}{2}\right)$

$$
K_{\mathcal{E}}^{\alpha}(u, x):=e^{\alpha u} k_{\mathcal{E}}^{\lambda(\alpha)}(x), \quad(u, x) \in \Sigma \times \mathbb{R} .
$$

Here $\lambda(\alpha)=\alpha^{2}+(d-2) \alpha$ (thus $\left.\lambda\left(\alpha_{0}\right)=-(d-2)^{2} / 4\right)$. Recall $\boldsymbol{\Delta}$ denotes the Martin boundary of $\mathcal{C}_{Y}(\Sigma)$ w.r. to $L$, and $\boldsymbol{\Delta}_{1}$ its minimal part.

Theorem 6.7. The function $K_{\mathcal{E}}^{\alpha_{0}}$ is L-minimal (so $K_{\mathcal{E}}^{\alpha_{0}}=K_{\xi}$ for some $\xi \in \boldsymbol{\Delta}_{1}$ ) and there exists $\Phi: \Sigma \rightarrow \mathbb{R}_{+}$going to $+\infty$ along $\mathcal{E}$ and such that $\left(u_{j}, y_{j}\right) \rightarrow \xi$ when $\left|u_{j}\right| \leq \Phi\left(y_{j}\right)$ and $\left\{y_{j}\right\} \rightarrow \mathcal{E}$. Moreover for every $\alpha \in\left(\alpha_{0}, \alpha_{\max }\right), K_{\mathcal{E}}^{\alpha}$ is minimal L-harmonic in $\mathcal{C}_{y}(\Sigma)$ and each associated sequence $\left\{\left(u_{j}, y_{j}\right)\right\}$ in $\mathcal{C}_{Y}(\Sigma)$ satisfies: (i) $\left\{y_{j}\right\} \rightarrow \mathcal{E}$ and (ii) $u_{j} \rightarrow+\infty$.

Similarly, for $\alpha \in\left(\alpha_{\min }, \alpha_{0}\right)$, the function $K_{\mathcal{E}}^{\alpha}$ is $L$-minimal in $\mathcal{C}_{y}(\Sigma)$ and every associated sequence $\left\{u_{j}, y_{j}\right\}$ satisfies: (i) $\left\{y_{j}\right\}$ converges to $\mathcal{E}$ and (ii) $u_{j} \rightarrow-\infty$.

Proof. (a) By Lemma 6.6, if $\left\{y_{j}\right\}$ is a sequence in $\Sigma$ converging towards $\mathcal{E}$ and such that $\left\{\left(0, y_{j}\right)\right\}$ converges to some $\xi \in \boldsymbol{\Delta}$, the Martin function $K_{\xi}$ satisfies: $K_{\xi}(\rho, x) / K_{\xi}\left(\rho^{\prime}, x\right)=e^{-\frac{d-2}{2}\left(\rho-\rho^{\prime}\right)}$ for $\rho, \rho^{\prime} \in \mathbb{R}$. Thus $K_{\xi}(u, x)=e^{-\frac{d-2}{2} u} k(x)$ where $k$ is independent of $u$ and necessarily a positive solution of $\Delta_{S} k+\lambda_{0} k=0$ in $\Sigma, \lambda_{0}=\lambda\left(\alpha_{0}\right)$.

Using the John cuts $M_{n}$ (the mediator in $B_{n}$ between $T_{n}$ and $T_{n}^{\prime}$ ), $n \geq 1$, and Proposition 4.2 we see that $K_{\xi}$ vanishes on $\tilde{\partial} \Sigma \times \mathbb{R}$. So $k=0$ in $\tilde{\partial} \Sigma$ and as mentioned before $k$ must be the $\left(\Delta_{S_{d-1}}+\lambda_{0} I\right)$-minimal function corresponding to $\mathcal{E}$, i.e $k=k_{\mathcal{E}}^{\lambda\left(\alpha_{0}\right)}$. 
(b) It follows that $\left(u_{j}, y_{j}\right) \rightarrow \xi$ when $\left\{u_{j}\right\}$ is bounded and $\left\{y_{j}\right\}$ is as before (see Proposition 2.12). And for $\rho_{y}$ growing sufficiently slowly to $+\infty$ as $y \rightarrow \mathcal{E}$, the point $(u, y)$ tends to $\xi$ for $y \rightarrow \mathcal{E}$ and $|u| \leq \rho_{y}$ (the convergence holds in the Martin space of $\left.\left(\mathcal{C}_{Y}(\Sigma), L\right)\right)$. In particular there is no minimal boundary point $\zeta=\lim \left(u_{j}, y_{j}\right)$, with $y_{j} \rightarrow \mathcal{E}$ of the finite type (i.e. non exponential in the vertical variable) described in Proposition 2.12 (iii).

(c) We now show that $K_{\xi}$ is minimal $L$-harmonic in $\mathcal{C}_{Y}(\Sigma)$ and more generally that for each $\alpha \in\left[\alpha_{0}, \alpha_{\max }\right)$ the function $h_{0}(u, x)=e^{\alpha u} k_{0}(x)$, where $k_{0}=k_{\mathcal{E}}^{\lambda(\alpha)}$ is minimal harmonic for $\left(\mathcal{C}_{Y}(\Sigma), L\right)$. Consider its Martin's disintegration into $L$-minimal functions. This disintegration does not charge the set of minimal functions in the form $e^{\beta u} k(x)$ with $\beta \neq \alpha$ (this would contradict the behavior of $h_{0}$ as $u \uparrow+\infty$ or $u \downarrow-\infty)$. Since $h_{0}=0$ on $\mathbb{R} \times \widetilde{\partial} \Sigma$, it is supported by the set of minimal points $\zeta$ such that $\zeta=\lim \left(u_{j}, y_{j}\right),\left|u_{j}\right| \rightarrow \infty$ and $y_{j} \rightarrow \mathcal{E}$ (using Proposition 4.2 and (b) above) and hence $K_{\zeta}(u, x)=e^{\beta u} k(x)$ with $k$ vanishing on $\tilde{\partial} \Sigma$. Thus $k=k_{\mathcal{E}}^{\lambda(\beta)}$ and the disintegration is supported by a minimal point $\zeta$ such that $K_{\zeta}=h_{0}$.

The remaining assertion clearly follows from Corollary 4.4 and the proof is complete.

\subsection{Proof of Theorem 4.6 and a second example}

In this section we construct a second example - based on the first - for which there are, for each $\alpha \in\left(\alpha_{\min }, \alpha_{\max }\right)$, corresponding minimal and non minimal Martin points $\zeta$ with $K_{\zeta}(u, x)=e^{\alpha u} k(x)$. We first establish - in the spirit of [2]Theorem 4.6.

A. Preliminaries. Denote $\Sigma_{+}$the hemisphere $\left\{t \in S_{d-1} ; t_{1}>0\right\}$ of $S_{d-1}$, $x_{0}=(1,0, \ldots, 0)$ its center, and $\sigma$ the reflexion $\left(t_{1}, \ldots, t_{d}\right) \mapsto\left(-t_{1}, t_{2}, \ldots, t_{d}\right)$.

Proposition 6.8. Assume that $\Sigma \supset \Sigma_{+}$and denote $G$ the Green's function of $\mathbb{R} \times \Sigma$. Given $\rho>0$, there is a $C=C(d, \rho)$ such that whenever $y \in \Sigma_{+}, x \in \Sigma$, and $u \leq v-2 \rho$

$$
G_{(v, y)}(u, x):=G(u, x ; v, y) \leq C G_{(v, y)}\left(u, x_{0}\right) .
$$

Proof. a) By a known general estimate (see [2], [4]) of the Green's function of a domain containing a $C^{2}$-ball (here $\left\{(t, x) \in \mathcal{C}_{Y}(\Sigma) ;\left|x-x_{0}\right|^{2}+|t-w|^{2} \leq 2\right\}$ ) - together with Harnack inequalities and elementary observations - we have when $z \in \Sigma_{+}, z^{\prime} \in \Sigma,\left|w^{\prime}-w\right| \geq \rho:$

$$
G\left(w^{\prime}, z^{\prime} ; w, z\right) \leq C_{d, \rho} G\left(w \pm \rho, x_{0} ; w, z\right) .
$$

b) Denote $\tilde{\sigma}(t, x)=(t, \sigma(x))$. Applying the maximum principle in $\mathbb{R} \times \Sigma_{+}$ to the functions $G_{(v, y)}$ and $G_{(v, y)} \circ \tilde{\sigma}$ in $\mathcal{C}_{Y}\left(\sigma\left(\Sigma_{+}\right)\right)$-extend the second by zero outside $\mathcal{C}_{Y}(\sigma(\Sigma))$ - we have

$$
G_{(v, y)}(w, z) \leq G_{(v, y)}(w, \sigma(z)) \leq \sup \left\{G_{(v, y)}\left(w, z^{\prime}\right) ; z^{\prime} \in \Sigma_{+}\right\}
$$

for $z \in \Sigma_{-}=\Sigma \backslash \Sigma_{+}, w \neq v$. In particular we need only consider $x \in \Sigma_{+}($take $w=u)$. 
Similarly, if $\mu_{(u, x)}^{t}, t \in \mathbb{R}$, is the $L$-harmonic measure of $(u, x), u \leq t$, in the truncated cylinder $C_{-}^{t}:=\mathcal{C}_{Y}(\Sigma) \cap\{(w, z) ; w<t\}$ we have for $x \in \Sigma_{+}$

$$
\mu_{(u, x)}^{t} \leq \sigma\left(\mu_{(u, x)}^{t}\right) \quad \text { in }\{t\} \times \sigma\left(\Sigma_{-}\right) \subset \partial C_{-}^{t} .
$$

This is because the adjoint Green's function $\stackrel{*}{G}_{(u, x)}^{t}$ with respect to $C_{-}^{t}$ is larger $\operatorname{than} \stackrel{*}{G}_{(u, x)}^{t} \circ \tilde{\sigma}$ in $C_{-}^{t} \cap\left\{(w, z) ; z \in \Sigma_{+}\right\}$and $\mu_{(u, x)}^{t}(t, d z)$ is $\partial_{w} \stackrel{*}{(u, x)}_{(u)}^{*} d \sigma_{S}(z)$.

c) Now write the réduite (w. r. to the cylinder $\left.\mathcal{C}_{Y}(\Sigma)\right)$ of $G_{(v, y)}$ over $\Sigma_{+}^{u+\rho}:=$ $\{u+\rho\} \times \Sigma_{+}$, i.e., $p=R_{G_{(v, y)}}^{\Sigma_{+}^{u+\rho}}([11],[13])$, as a potential $G_{\mu}$ of a positive measure $\mu$ on $\Sigma_{+}^{u+\rho}$. Then

$$
\begin{aligned}
G_{\mu}(u, x) & =\int_{\Sigma_{+}^{u+\rho}} G(u, x ; \zeta) d \mu(\zeta) \leq C \int_{\Sigma_{+}^{u+\rho}} G\left(u, x_{0} ; \zeta\right) d \mu(\zeta) \\
& =C G_{\mu}\left(u, x_{0}\right) \leq C G_{(v, y)}\left(u, x_{0}\right),
\end{aligned}
$$

using (6.10).

d) Finally $q=G_{(v, y)}-p$ is majorized in $C_{-}^{u+\rho}$ by the solution $h$ to the Dirichlet problem in $C_{-}^{u+\rho}$, with the boundary condition $h=G_{(v, y)}$ in $\Sigma_{-} \times\{u+\rho\}$ and $h=0$ on the rest of $\partial U$. By (6.11) and (6.12), $q(u, x) \leq p(u, x)$ and by $(6.13)$ $q(u, x) \leq C G_{(v, y)}\left(u, x_{0}\right)$.

Corollary 6.9. Suppose $\Sigma_{+} \subset \Sigma$. Let $\left\{y_{j}\right\}$ be a sequence in $\Sigma^{+}$and let $v_{j} \rightarrow+\infty$ in $\mathbb{R}$. Then $\left\{\left(v_{j}, y_{j}\right)\right\}$ converges to the canonical Martin point at $+\infty$, i.e.,

$$
\lim _{j} K_{\left(v_{j}, y_{j}\right)}=F_{+} .
$$

This is Theorem 4.6. Similarly of course $\lim _{j} K_{\left(v_{j}, y_{j}\right)}=F_{-}$, when $v_{j} \rightarrow-\infty$ with $y_{j} \in \Sigma_{+}$.

Proof. If $\zeta$ is a cluster Martin point for $\left\{\left(v_{j}, y_{j}\right)\right\}_{j \geq 1}$ it immediately follows from Proposition 6.8 that if $h$ denotes the $L$-harmonic measure of $\{0\} \times \Sigma$ in $\mathcal{C}_{Y}(\Sigma)$,

$$
K_{\zeta}(u, x) \leq C K_{\zeta}\left(u_{0}, x_{0}\right) h\left(u-u_{0}, x\right)
$$

for $u \leq u_{0}, x \in \Sigma$. This shows that $K_{\zeta}$ vanishes on $\mathbb{R} \times \partial \Sigma$ and that $K_{\zeta}$ is bounded for $u \leq u_{0}$. Thus $K_{\zeta}=F_{+}$by Theorem 2.11 .

B. Assuming $d \geq 4$ we construct $\Sigma$ as follows. We start with the hemisphere $\Sigma_{+}$, a point $P_{0} \in \partial \Sigma_{+}$and a sequence of points $P_{n} \in \partial \Sigma_{+}$such that $\left|P_{n}-P_{0}\right|=4^{-n}, n \geq 1$. For each $n \geq 1$, let $\Omega_{n}$ be a domain in $S_{d-1}, \bar{\Omega}_{n} \subset$ $B_{S_{d-1}}\left(P_{n}, \frac{1}{4^{n+1}}\right) \backslash \bar{\Sigma}_{+}$of the type considered in Remark 6.3, (starting with disjoint balls $\bar{B}_{n, j}, j \geq 1$, in $S_{d-1}$ converging to some point $Q_{n}$ in $B\left(P_{n}, \frac{1}{4^{n+1}}\right) \backslash \bar{\Sigma}_{+}$and such that the sum of the squares of their radii diverges). Let $U_{n}$ be a region in $B\left(P_{n}, \frac{1}{4^{n+1}}\right) \backslash\left(\bar{\Sigma}_{+} \cup \bar{\Omega}_{n}\right)$ such that $\bar{U}_{n} \cap \bar{\Sigma}_{+}$is a closed ball $\bar{\Delta}_{n}$ in $\partial \Sigma_{+}$of center $P_{n}$ and $\bar{U}_{n} \cap \bar{\Omega}_{n}$ a cap $\bar{\Delta}_{n}^{\prime} \subset \partial B_{n, 1} \cap \partial \Omega_{n}$ (where $\Delta_{n}$ and $\Delta_{n}^{\prime}$ are the relative interiors). 
The domain $\Sigma$ is the union of $\Sigma_{+}$, the joining regions $U_{n}$ and the disks $\Delta_{n}$ and $\Delta_{n}^{\prime}, n \geq 1$.

Let $\alpha \in\left(\alpha_{\min }, \alpha_{\max }\right)$ and let $n \geq 1$. As before, by [4] there is a unique positive $(\Delta+\lambda(\alpha) I)$-harmonic function $k_{n}$ in $\Sigma$ vanishing in $\partial \Sigma \backslash\left\{Q_{n}\right\}$ and such that $k_{n}\left(x_{0}\right)=1\left(x_{0}\right.$ is the center of the hemisphere $\left.\Sigma_{+}\right)$. Moreover $k_{n}$ is minimal $\Delta$-harmonic in $\Sigma$ and by Theorem 6.7 the function $K_{Q_{n}}^{(\alpha)}(u, x)=e^{\alpha u} k_{n}(x)$ is minimal $L$-harmonic in $\mathcal{C}_{Y}(\Sigma)$. Denote $h^{(\alpha)}(u, x)=e^{\alpha u} k(x)$ the similar $L$-harmonic function in $\mathcal{C}_{Y}(\Sigma)$ with a pole at $P_{0}$.

Proposition 6.10. The function $h^{(\alpha)}$ is a non minimal Martin function for $\left(\mathcal{C}_{Y}(\Sigma), L\right)$.

Proof. It follows from standard arguments that the $k_{n}$ vanish uniformly on the boundary of $\Sigma$ away from $P_{0}($ as $n \rightarrow \infty)$, so that $k=\lim k_{n}$. Thus $h^{(\alpha)}=\lim K_{Q_{n}}^{(\alpha)}$ and $h^{(\alpha)}$ is an $L$-Martin function in $\mathcal{C}_{Y}(\Sigma)$ associated to a point $\zeta \in \boldsymbol{\Delta}$. If $\alpha \geq \alpha_{0}$ (resp. $\left.\alpha \leq \alpha_{0}\right)$, there is a sequence $\left\{\left(v_{j}, y_{j}\right)\right\}$ with $y_{j} \in \Omega_{j}, \lim v_{j}=+\infty$ (resp. $\left.\lim v_{j}=-\infty\right)$ converging to $\zeta$.

By Corollary 6.9, the point $\zeta$-as a Martin boundary point - is not in the closure of $\mathbb{R} \times \Sigma_{+}$. Thus every sufficiently small neighborhood $V$ of $\zeta$ meet $\mathbb{R} \times \Omega_{n}$ for all large $n$, but not $\mathbb{R} \times \Sigma_{+}$. And $V \cap \mathcal{C}_{Y}(\Sigma)$ is not connected. But (by a general property) each neighborhood of a minimal Martin boundary point contains another whose trace in $\mathcal{C}_{Y}(\Sigma)$ is connected (see, e.g., page 223 of [31]). Hence $\zeta$ is not minimal.

\section{Extensions to more general cylinders}

The argument in Sections 2 and 3 can be extended to more general second order elliptic operators in cylinders. We describe here a simple generalization and state the corresponding results. Assume $\Sigma$ is a relatively compact region in a $C^{1}$ Riemannian manifold $M$ of dimension $d-1(d \geq 2)$ equipped with a second order elliptic operator $L_{M}$ in the form

$$
L_{M}(\varphi)=\operatorname{div}(A \nabla \varphi)+B \cdot \nabla \varphi+\gamma \varphi
$$

where $A$ is a measurable, bounded and uniformly elliptic section of $\operatorname{End}(T(M)), B$ a bounded measurable vector field in $M$ and $\gamma$ a nonpositive bounded measurable function in $M$ (ref. [36]). We also assume that $M \backslash \Sigma$ is non polar. Thus $L_{M}$ admits a Green function in $\Sigma$.

We consider now a differential operator in the cylinder $\mathcal{C}_{Y}(\Sigma)=\mathbb{R} \times \Sigma$ which is a direct sum $L=L_{\mathbb{R}} \oplus L_{M}$ where $L_{\mathbb{R}}=\frac{d^{2}}{d u^{2}}+b \frac{d}{d u}$ is translation invariant in $\mathbb{R}$ (i.e., $b$ is a real constant). Again we fix some $x_{0} \in \Sigma$ and take $\left(0, x_{0}\right)$ as the normalization point in $\mathcal{C}_{Y}(\Sigma)$ for the Martin functions.

We define $\lambda_{1}:=\lambda_{1}\left(L_{M} ; \Sigma\right)$ as the supremum of all real $t$ such that $L_{M}+t I$ admits a Green's function in $\Sigma$ (or such that the cone of nonnegative $L_{M}+t I$ superharmonic functions has a dimension $>1$ ). It is well known that $0<\lambda_{1}<\infty$ 
and that for $t:=\lambda_{1}$ all nonnegative $\left(L_{M}+t I\right)$-superharmonic functions in $\Sigma$ are proportional to the unique (up to scalar multiplication) positive $L_{M}+t I$ solution $\varphi_{0}$ in $\Sigma$. This solution $\varphi_{0}$ is bounded, vanishes in the weak sense on the boundary $\partial \Sigma$ and $\varphi_{0} \in H_{0}^{1}(\Sigma)$. As also well-known we have similar properties for the formal adjoint operator $L_{M}^{*}$, and moreover $\lambda_{1}\left(L_{M}^{*} ; \Sigma\right)=\lambda_{1}\left(L_{M} ; \Sigma\right)$. We denote $\varphi_{0}^{*}$ a positive $\left(L^{*}+\lambda_{1} I\right)$-superharmonic function in $\Sigma$. Again $\varphi_{0}^{*}$ is unique up to multiplication by a constant, vanishes on $\partial \Sigma$ and is $\left(L_{M}^{*}+\lambda_{1} I\right)$-harmonic in $\Sigma$.

Repeating the argument used in Section 2 we obtain a similar description of the $L$-minimal Martin function associated with the end $u \rightarrow+\infty$ in $\mathcal{C}_{Y}(\Sigma)$.

Proposition 7.1. If $K$ is a minimal L-Martin function in $\mathcal{C}_{Y}(\Sigma)=\mathbb{R} \times \Sigma$ associated to a sequence $\left(u_{j}, x_{j}\right)$ with $u_{j} \rightarrow+\infty, x_{j} \in \Sigma$, then $K$ is in the form

$$
K(u, x)=e^{\alpha u} K(0, x), \quad(u, x) \in \mathbb{R} \times \Sigma
$$

for some $\alpha \geq-\frac{b}{2}$ and $s(x)=K(0, x)$ is a proper function in $\Sigma: L_{M} s+\lambda s=0$, $\lambda=\alpha^{2}+b \alpha$. So $\alpha=\frac{-b+\sqrt{b^{2}+4 \lambda}}{2}$ and $-\frac{b^{2}}{4} \leq \lambda \leq \lambda_{1}$; moreover $s$ is $\left(L_{M}+\lambda I\right)$ minimal in $\Sigma$.

As before there is a natural bijection $K \mapsto \tilde{K}$ between the set $\boldsymbol{\Delta}^{+\infty}$ of the Martin function arising from some sequence $\left(v_{j}, y_{j}\right)$ in $\mathcal{C}_{Y}(\Sigma)$ with $\lim v_{j}=+\infty$ and the analogue set $\boldsymbol{\Delta}^{-\infty}$ (related to the condition $\lim v_{j}=-\infty$ ) by letting $\tilde{K}(u, x)=e^{-b u} K(-u, x)$.

Theorem 2.13 can also be extended to the present framework, but a slight modification is required in the proof. Set

$$
F_{+}(u, x)=e^{\alpha_{\max } u} \frac{\varphi_{0}(x)}{\varphi_{0}\left(x_{0}\right)} \quad \text { and } \quad F_{-}(u, x)=e^{\alpha_{\min } u} \frac{\varphi_{0}(x)}{\varphi_{0}\left(x_{0}\right)},
$$

where $\alpha_{\max }:=\frac{-b+\sqrt{b^{2}+4 \lambda_{1}}}{2}$ and $\alpha_{\min }:=\frac{-b-\sqrt{b^{2}+4 \lambda_{1}}}{2}$.

Theorem 7.2. If $f$ is L-harmonic in $\mathcal{C}_{Y}(\Sigma)$ and vanishes in weak sense on $\mathbb{R} \times \partial \Sigma$, then $f$ is a linear combination of $F_{+}$and $F_{-}$. In particular, if moreover $\liminf \operatorname{in}_{u \rightarrow-\infty} e^{\alpha_{\min } u} f\left(u, x_{0}\right)=0$ then $f$ is proportional to $F_{+}$. Thus $F_{+}$and $F_{-}$are L-minimal in $\mathcal{C}_{Y}(\Sigma)$.

Proof. As in the proof of Theorem 2.13, we may reduce ourselves to show the following. A function $F$ in $\mathcal{C}_{Y}(\Sigma)$ which vanishes on $\mathbb{R} \times \partial \Sigma$ and which is in the form $F(u, x)=\int_{A^{\prime}} K_{\zeta}(u, x) d \mu(\zeta)$ where $A^{\prime}=\left\{\zeta \in \boldsymbol{\Delta}^{+\infty} \cap \boldsymbol{\Delta}_{1} ;-\frac{b}{2} \leq \alpha(\zeta) \leq \alpha_{1}\right\}$, $\alpha_{1}<\alpha_{\max }$, and where $\mu$ is a finite positive Borel measure on $A$, must be the zero function. Denote $\lambda_{1}^{\prime}=\alpha_{1}^{2}+b \alpha_{1}$.

As before, the function $\varphi(x)=\int_{A^{\prime}} K_{\zeta}(0, x) d \mu(\zeta)$ is positive superharmonic with respect to $L_{0}=L_{M}+\lambda_{0} I, \lambda_{0}=-b^{2} / 4$ and vanishes in the weak sense on $\partial \Sigma$. The measure $-L_{0}(\varphi)$ is given by the density $\psi(x)=\int_{A}\left(\lambda-\lambda_{0}\right) k_{\zeta}^{\lambda}(x) d \mu(x)$. It follows that $\varphi$ is the $L_{0}$-Green's potential in $\Sigma$ of $\psi$ and again, $G_{\Sigma}^{L_{0}}(\psi)=\varphi \geq$ $\frac{1}{\lambda_{1}^{\prime}-\lambda_{0}} \psi$ in $\Sigma$, where $G_{\Sigma}^{L_{0}}$ is Green's function in $\Sigma$ w.r. to $L_{0}$. To conclude we 
then slightly modify the argument in Section 2 using now the minimal heat semigroup $P_{t}$ generated by $L$ in $\Sigma$ :

$$
\int_{\Sigma} G_{\Sigma}^{L_{0}}(\psi) \varphi_{0}^{*} d \sigma_{M}=\int_{0}^{+\infty} \int_{\Sigma} e^{\lambda_{0} t} P_{t}(\psi) \varphi_{0}^{*} d \sigma_{M} d t=\int_{0}^{+\infty} \int_{\Sigma} e^{-\left(\lambda_{1}-\lambda_{0}\right) t} \psi \varphi_{0}^{*} d \sigma d t
$$

because $P_{t}^{*}\left(\varphi_{0}\right)=e^{-\lambda_{1} t} \varphi_{0}^{*}$. Thus

$$
\int_{\Sigma} G_{\Sigma}^{L_{0}}(\psi) \varphi_{0}^{*} d \sigma_{M}=\frac{1}{\lambda_{1}-\lambda_{0}} \int \psi \varphi_{0}^{*} d \sigma_{M} .
$$

But on the other hand from $G_{\Sigma}^{L_{0}}(\psi) \geq \frac{1}{\lambda_{1}^{\prime}-\lambda_{0}} \psi$ it follows that $\int_{\Sigma} G_{\Sigma}^{L_{0}}(\psi) \varphi_{0}^{*} d \sigma_{M}$ is larger than $\frac{1}{\lambda_{1}^{\prime}-\lambda_{0}} \int \psi \varphi_{0}^{*} d \sigma$. Thus $\int \psi \varphi_{0}^{*} d \sigma=0$ and $\psi=0$ in $\Sigma$. So $\varphi=0$.

Corollary 7.3. Every positive L-harmonic function $f(u, x)$ on $\mathcal{C}_{Y}(\Sigma)$ vanishing (in the weak sense) on $\mathbb{R} \times \partial \Sigma$ and such that $\lim _{\sup _{u \rightarrow-\infty}} f\left(u, x_{0}\right)<\infty\left(x_{0} \in \Sigma\right)$ is proportional to $F_{+}$.

Theorems 3.1 and 5.1 extend as follows.

Theorem 7.4. Let $\xi_{j}=\left(v_{j}, y_{j}\right), j \geq 1$, be a sequence of points in $\mathcal{C}_{Y}(\Sigma)$ such that $v_{j} \rightarrow+\infty$. If $d \leq 3$ or if $d \geq 4$ and $\left\{y_{j}\right\}$ is relatively compact in $\Sigma$, the functions $K_{\xi_{j}}(u, x)$ converge to $K_{\zeta_{\infty}}(u, x):=e^{\alpha_{\Sigma} u} \psi_{0}(x)$. In particular when $d \leq 3, \zeta_{\infty}$ is the only Martin point at infinity.

The proofs are the same as above in Section 5 using the natural extensions of (5.1) (with $y$ in a relatively compact subset of $\Sigma$ when $d \geq 4$ ) to our present setting. Denote $\left\{\pi_{t}\right\}$ the heat semi-group generated by the $L$ in $\Sigma$ and as above $\varphi_{0}^{*}$ any positive eigenfunction of the adjoint elliptic operator $-L^{*}$ in $\Sigma$ for the eigenvalue $\lambda_{1}$. Then we have:

(i) if $d=3$, there is a $t_{0}>0$ and a function $C:\left[t_{0}, \infty\right) \rightarrow(1,+\infty)$ such that $\lim _{t \rightarrow \infty} C(t)=1$ and (if $C_{0}=\left(\int_{\Sigma} \varphi_{0}(y) \varphi_{0}^{*}(y) d \sigma(y)\right)^{-1}$ )

$$
C_{0} C(t)^{-1} e^{-\lambda_{1} t} \varphi_{0}\left(x_{0}\right) \varphi_{0}^{*}(y) \leq \pi_{t}\left(x_{0}, y\right) \leq C_{0} C(t) e^{-\lambda_{1} t} \varphi_{0}\left(x_{0}\right) \varphi_{0}^{*}(y), t \geq t_{0}
$$

for all $y \in \Sigma$. The proof of Theorem 1 in [10] for the Laplacian can be adapted after one shows that the Cranston-McConnell inequalities [14], [7] (see also [9]) hold for all subdomains $\omega$ of $\Sigma$ : i.e., there is a constant $C=C(\Sigma)$ such that for every $\omega$ and every positive $L$-harmonic function $h$ in $\omega$ one has $G^{\omega}(h) \leq C|\omega| h$.

(ii) For all $d \geq 3$, it is well-known that (7.3) holds provided $y$ is restricted to a relatively compact subset $A$ of $\Sigma$ (see Theorem 1.2 (iii) in [32] with a class of elliptic operators slightly different from ours; see also [33], [34]).

Let us finally also mention that the results in Sections 4.1 and 4.2 extend to the present setting if we restrict to John conditions with $N=1$, where $N$ is the number of poles - recall the needed results in [6] require for $N \geq 2$ the symmetry of the underlying operator. When $B=0$ and $A$ is symmetric, the restriction $N=1$ can be removed since $L=L_{\mathbb{R}}+\partial_{u u}^{2}+b \partial_{u}$ is then symmetric with respect to the reference measure $\mu(d u, d x)=e^{b u} d u d \sigma_{M}(x)$ (i.e. $L$ is symmetric in $M \times \mathbb{R}$ equipped with the Riemannian metric $\left.g_{(u, x)}(d u, d x)=e^{b u}(d u)^{2} g_{M}(d x)\right)$. 


\section{References}

[1] Aikawa, H., Hirata, K. And Lundh, T.: Martin boundary points of a John domain and unions of convex sets. J. Math. Soc. Japan 58 (2006), no. 1, 247-274.

[2] Ancona, A.: Une propriété de la compactification de Martin d'un domaine euclidien. Ann. Inst. Fourier (Grenoble) 29 (1979), no. 4, 71-90.

[3] Ancona, A.: Negatively curved manifolds, elliptic operators and the Martin boundary. Ann. of Math. (2) 125 (1987), no. 3, 495-536.

[4] Ancona, A.: Régularité d'accès des bouts et frontière de Martin d'un domaine euclidien. J. Math. Pures Appl. (9) 63 (1984), no. 2, 215-260.

[5] Ancona, A.: Théorie du Potentiel sur les graphes et les variétés. In Ecole d'été de Probabilités de Saint-Flour XVIII-1988, 1-112. Lecture Notes in Math. 1427, Springer, Berlin, 1990.

[6] Ancona, A.: Sur la théorie du potentiel dans les domaines de John. Publ. Mat. 51 (2007), no. 2, 345-396.

[7] Bañuelos, R.: On an estimate of Cranston and McConnell for elliptic diffusions in uniform domains. Probab. Theory Related Fields 76 (1987), no. 3, 311-323.

[8] BaÑuelos, R.: Intrinsic ultracontractivity and eigenfunctions estimates for Schrödinger operators. J. Funct. Anal. 100 (1991), no. 1, 181-206.

[9] Bañuelos, R.: Lifetime and heat kernel estimates in nonsmooth domains. In Partial differential equations with minimal smoothness and applications (Chicago, 1990), 37-48. IMA Vol. Math. Appl. 42, Springer, New York, 1992.

[10] Bañuelos, R. AND DAvis, B.: Heat kernel, eigenfunctions and conditioned Brownian motion in planar domains. J. Funct. Anal. 84 (1989), no. 1, 188-200.

[11] Brelot, M.: Axiomatique des fonctions harmoniques. Séminaire de Mathématiques Supérieures 14, Les Presses de l'Université de Montréal, Montreal, Que., 1969.

[12] Chavel, I. And Karp, L.: Large time behavior of the heat kernel: the parabolic $\lambda$-potential alternative. Comment. Math. Helv. 66 (1991), no. 4, 541-556.

[13] Constantinescu, C. And Cornea, A.: Potential theory on harmonic spaces. Die Grundlehren der mathematischen Wissenschaften 158, Springer-Verlag, New YorkHeidelberg, 1972.

[14] Cranston, M. and McConnell, T. R.: The lifetime of conditioned Brownian motion. Z. Wahrsch. Verw. Gebiete 65 (1983), no. 1, 1-11.

[15] Davies, E. B. AND Simon, B.: Ultracontractivity and the heat kernel for Schrödinger operators and Dirichlet Laplacians. J. Funct. Anal. 59 (1984), no. 2, 335-395.

[16] Deny, J. And Lelong, P.: Étude des fonctions sousharmoniques dans un cylindre ou dans un cône. Bull. Soc. Math. France 75 (1947), 89-112.

[17] Doob, J. L.: Classical potential theory and its probabilistic counterpart. Classics in Mathematics, Springer-Verlag, Berlin, 2001.

[18] Fabes, E. B., Garofalo, N. and Salsa, S.: A backward Harnack inequality and Fatou theorem for nonnegative solutions of parabolic equations. Illinois J. Math. 30 (1986), no. 4, 536-565.

[19] Freire, A.: On the Martin boundary of Riemannian products. J. Differential Geom. 33 (1991), no. 1, 215-232. 
[20] Gesztesy, F. And Zhao, Z.: Domain perturbations, Brownian motion, capacities and ground states of Dirichlet-Schrödinger operators. Math. Z. 215 (1994), no. 1, $143-150$.

[21] Guivarc'H, Y., Ji, L. And TAYlor, J. C.: Compactifications of symmetric spaces. Progress in Mathematics 156, Birkhäuser, Boston, MA, 1998.

[22] Heurteaux, Y.: Solutions positives et mesure harmonique pour des opérateurs paraboliques dans des ouverts "lipschitziens". Ann. Inst. Fourier (Grenoble) $4 \mathbf{1}$ (1991), no. 3, 601-649.

[23] Hirata, K.: Martin Boundary points of cones generated by spherical John regions. Ann. Acad. Sci. Fenn. Math. 32 (2007), no. 2, 289-300.

[24] Levin, B. YA. And Kheyfits, A. I.: Asymptotic behavior of subfunctions of the stationary Schrödinger operator. arXiv:math/0211328v1 (2002). See also: Some topics on value distribution and differentiability in complex and p-adic analysis, 323-397. Math. Monogr. Ser. 11, Sci. Press, Beijing, 2008.

[25] Kuran, Ü.: On NTA-conical domains. J. London Math. Soc. (2) 40 (1989), no. 3, $467-475$.

[26] Martin, R. S.: Minimal positive harmonic functions. Trans. Amer. Math. Soc. 49 (1941), 137-172.

[27] Molčanov, S. A.: The Martin boundary of a direct product of Markov processes. Sibirsk. Mat. Ž. 11 (1970), 370-380.

[28] Moser, J.: On a pointwise estimate for parabolic differential equations. Comm. Pure Appl. Math. 24 (1971), 727-740.

[29] Murata, M.: On construction of Martin boundaries for second order elliptic equations. Publ. Res. Inst. Math. Sci. 26 (1990), no. 4, 585-627.

[30] Murata, M.: Martin boundaries of elliptic skew products, semismall perturbations and fundamental solutions of parabolic equations. J. Funct. Anal. 194 (2002), no. 1, $53-141$.

[31] NAÏM, L.: Sur le rôle de la frontière de R.S. Martin dans la théorie du potentiel. Ann. Inst. Fourier, Grenoble 7 (1957), 183-281

[32] Pinchover, Y.: Large time behavior of the heat kernel and the behavior of the Green function near criticality for nonsymmetric elliptic operators. J. Funct. Anal. 104 (1992), no. 1, 54-70.

[33] Pinchover, Y.: Large time behavior of the heat kernel. J. Funct. Anal. 206 (2004), no. 1, 191-209.

[34] Pinsky, R. G.: The lifetimes of conditioned diffusion processes. Ann. Inst. H. Poincaré Probab. Statist. 26 (1990), no. 1, 87-99.

[35] Rashkovskiř, A. Yu. and Ronkin, L. I.: Subharmonic functions of finite order in a cone. III. Functions of completely regular growth. J. Math. Sci. 77 (1995), no. 1, 2929-2940.

[36] Stampacchia, G.: Le problème de Dirichlet pour les équations elliptiques du second ordre à coefficients discontinus. Ann. Inst. Fourier (Grenoble) 15 (1965), no. 1, $189-258$.

[37] Yoshida, H. and Miyamoto, I.: Solutions of the Dirichlet problem on a cone with continuous data. J. Math. Soc. Japan 50 (1998), no. 1, 71-93.

[38] Yoshida, H. And Miyamoto, I.: Harmonic functions in a cone which vanish on the boundary. Math. Nachr. 202 (1999), 177-187. 
Received March 29, 2010.

Alano Ancona: Département de Mathématiques, Bâtiment 425, Université ParisSud 11, 91405 Orsay, France.

E-mail: alano.ancona@math.u-psud.fr 